\title{
EL PATRÓN COMERCIAL A FINALES DEL SIGLO XIX: UN ANÁLISIS COMPARATIVO
}

ANTONI ESTEVADEORDAL *

Banco Interamericano de Desarrollo

\section{RESUMEN}

En este ensayo se contrasta para una muestra de 18 paises hacia 1913 una versión estática de la teoría de Heckscher-Ohlin, en la formulación de Vanek. La conclusión alcanzada es que si bien el modelo de $\mathrm{H}$-O con competencia perfecta no resulta plenamente explicativo para la segunda posguerra mundial, es muy adecuado, sin embargo, para los años finales del siglo XXX y primeros del siglo $\mathrm{XX}$.

This paper will focus on the question does the Heckscher-Ohlin theory adequately account for the major aspects of the trade pattern observed prior to WWI, or are there other influences that mattered? I conclude that although the perfect competition $\mathrm{H}-\mathrm{O}$ model does not do all that well for the post-world War II period, it is perfectly adequate for the late nineteenth century, the period which motivated Eli F. Heckscher and Bertil Ohlin in the first place.

\section{INTRODUCCION}

Entre 1870 y 1913 la economía mundial experimentó una de las redistribuciones más espectaculares de los recursos productivos de la historia. La

* Economista en la División de Integración, Comercio y Asuntos Hemisféricos del Banco In. teramericano de Desarrollo, Washington, D.C. Este articulo está basado en un capitulo de la tesis teramericano de Desarrollo, Washington, D.C. Este articulo está basa la Universidad de Harvard.
doctoral del autor presentada en el Departamento de Economia de la
El autor agradece el constante apoyo y estimulo de Jeffrey G. Williamson, así como los comentarios de Richard N. Cooper, Bradford De Long y Claudia Goldin. 
principal causa de esta asombrosa redistribución fue la nueva tecnología del transporte introducida por la segunda oleada de la revolución industrial. La reducción de los costes del transporte terrestre y marítimo, asi como las mejoras de las comunicaciones, abrieron al comercio internacional regiones enteramente nuevas de América, Rusia, Oceanía y África. La migración de trabajo y capital a las regiones de "reciente colonización» que se registró durante este periodo fue la mayor que ha experimentado el mundo. En 1850, algunas zonas del este y sur de Europa aún parecian tener abundante tierra agrícola; pero estas regiones ya no podian competir con las extensiones de tierra fértil que los ferrocarriles y los barcos de vapor abrieron a los mercados mundiales a partir de 1870. La conjunción de estos cambios de las dotaciones relativas de recursos naturales y las diferencias entre las tasas de acumulación de capital humano y no humano de los distintos países alteró la pauta de especialización internacional del siglo XIX, lo que fue acompañado de un cambio de la relación de fuerzas política y económica. La posición única de que gozaba Gran Bretaña desde comienzos del siglo XIX comenzó a decaer ante la competencia alemana y americana ${ }^{1}$.

Una manera de examinar este cambio es comparar las proporciones del comercio mundial de bienes manufacturados correspondientes a cada país, como hizo H. Tyszynski en su estudio pionero del periodo 1899-1950 Tyszynski ${ }^{2}$. Las tendencias que describe Tyszynski en su trabajo son esencialmente las mismas que describe Maizels ${ }^{3}$ en el caso del periodo $1880-1913$ y que reproducimos aquí en el cuadro 1.1. El auge de Alemania y de Estados Unidos y el declive del Reino Unido son perfectamente conocidos. Pero igualmente espectacular es el declive de Austria y Francia y el auge más modesto de Canadá, Japón y Suecia, que ha sido objeto de una atención mucho menor.

A un economista le gustaría analizar estos cambios de la pauta de la ventaja comparativa en función de los cambios de la distribución internacional de los recursos utilizando una versión dinámica del modelo de comercio de Heckscher-Ohlin (H-O) que tanto se ha enseñado ${ }^{4}$. Sin embargo, en este ensayo, nos limitamos a contrastar una versión estática o a largo plazo de la proposición de H-O para el periodo anterior a la Primera Guerra Mundial, a saber,

1 En ningün sector era esto más importante que en la siderurgia. En 1913, las exportaciones alemanas de hierro y acero eran superiores a las britanicas y las americanas no le iban a la zaga; Gran Bretaña se habia convertido en un importante importador de acero (Allen, 1979; Milward y Saul, 1977).

2 Tyszynski (1951).

${ }^{3}$ Maizels (1963).

4 Bowen (1983) ha contrastado una versión dinámica del modelo H.O en el caso de Estados Unidos y del periodo 1963-1975. 
que el comercio internacional dependía fundamentalmente de las existencias relativas de recursos de los países. Utilizando la formulación del modelo de Vanek, ${ }^{5}$ en este artículo centramos la atención en la siguiente pregunta: ¿explica la teoria de Heckscher y Ohlin correctamente los principales aspectos de la pauta del comercio observada antes de la Primera Guerra Mundial o hay otros factores importantes? Llegamos a la conclusión de que aunque el modelo de $\mathrm{H}-\mathrm{O}$ con competencia perfecta no funciona muy bien en lo que se refiere al periodo posterior a la Segunda Guerra Mundial, es perfectamente adecuado para finales del siglo XIX, periodo que fue precisamente el que motivó en su momento a Eli F. Heckscher y Bertil Ohlin 6.

\section{CUADRO 1.1}

Proporción del comercio mundial correspondiente a los bienes manufacturados (1880-1913)

\begin{tabular}{|c|c|c|c|}
\hline País exportador & 1880 & 1899 & 1913 \\
\hline Estados Unidos . . . . . . . & n.a. & 11,7 & 13,0 \\
\hline Alemania ............... & 19,3 & 22,4 & 26,6 \\
\hline $\begin{array}{l}\text { Alemania ..... } \\
\text { Reino Unido . }\end{array}$ & 41,4 & 33,2 & 30,2 \\
\hline Francia ........ & 22,2 & 14,4 & 12,1 \\
\hline Japón $\ldots \ldots \ldots$ & n.a. & 1,5 & 2,3 \\
\hline Bélgica $. . . \ldots . .$. & 5,0 & 5,5 & 5,0 \\
\hline Canada . & n.a. & 0,4 & 0,6 \\
\hline Italia $\ldots$ & n.a. & 3,6 & 3,3 \\
\hline$\ldots \ldots \ldots, \ldots, \ldots, \ldots, \ldots, \ldots$ & n.a. & 4,0 & 3,1 \\
\hline 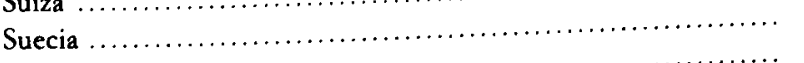 & 0,8 & 0,9 & 1,4 \\
\hline 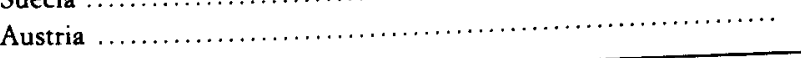 & 8,0 & n.a. & 5,0 \\
\hline
\end{tabular}

n.a. $=$ n.d.

FueNTES: A. Maizels (1963, p. 189) y A. S. Milward y S. B. Saul (1977, p. 473).

La estructura del resto del capítulo es la siguiente. En el apartado II pasamos revista a la literatura empírica moderna sobre el modelo de H-O. En el III pasamos revista a la proposición de $\mathrm{H}$-O en la literatura de historia econó-

5 Véase el apartado II de este artículo. En todo este capítulo nos referimos al modelo o bien con el nombre de Heckscher-Ohlin (H-O), o bien con el nombre de modelo Heckscher-OhlinVanek (H-O-V).

6 Para una nueva traducción inglesa de los escritos de Heckscher y Ohlin, véase Flam y Flanders (1991). 
mica. En el IV contrastamos una versión de la teoría de H-O para 1913 con una muestra de 18 países y con una base de datos recién construida. En el $\mathrm{V}$ utilizamos una versión ampliada del modelo para analizar distintas maneras de detectar el proteccionismo. En el VI exploramos las medidas comerciales $\mathrm{e}$ industriales distorsionadoras de terceros países cuando se analizan con el modelo estimado, poniendo énfasis en España e Italia. El apéndice contiene un análisis de los métodos y las fuentes utilizadas para elaborar los datos.

\section{CONTRASTE DEL MODELO DE HECKSCHER Y OHLIN: EVIDENCIA MODERNA}

Según el modelo de Heckscher y Ohlin, un país exporta la mercancía que utiliza intensivamente su factor relativamente abundante e importa la mercancía que utiliza intensivamente su factor relativamente escaso. El teorema se basa en los siguientes supuestos ${ }^{7}$ : (1) gustos homotéticos idénticos, (2) rendimientos constantes de escala y tecnologías idénticas, (3) competencia perfecta en los mercados de bienes y de factores, (4) intercambio internacional de mercancías sin costes, (5) factores de producción internacionalmente inmóviles que pueden desplazarse sin costes de unas industrias a otras dentro de un mismo país, (6) igual número de bienes que de factores (modelo cuadrado) y (7) suficientes similitudes en las dotaciones de factores para que los paises se encuentren en el mismo «cono de diversificación». Estos supuestos implican que los factores tienen los mismos precios relativos en todos los países (igualación de los precios de los factores) y que los cocientes entre los factores y la producción son idénticos. Estos supuestos también implican que el vector de exportaciones netas es una función lineal del vector de dotaciones de factores.

El modelo que nos proponemos aplicar aqui es una representación en forma reducida de la pauta de comercio de un país basada en la tecnologia existente y en las dotaciones relativas de factores. La producción $Q$ de un pais se obtiene a partir de una matriz de uso de los factores $A$ y un conjunto de dotaciones $V$,

$$
Q=A^{-1} V
$$

\footnotetext{
7 Para una sencilla exposición, véase Caves, Frankel y Jones (1990). Para análisis más avanzados, véase Dixit y Norman (1980) y Leamer (1984).
} 
La producción mundial puede describirse de la misma manera:

$$
Q_{w}=A^{-1} V_{w}
$$

Suponiendo que las funciones de utilidad son homotéticas e idénticas y los precios de los factores son iguales, cada pais consume cada variedad de bienes en la misma proporción:

$$
C=s Q_{w}
$$

donde $s$ es la proporción de la producción mundial que consume el país y $\mathrm{C}$ es su vector de consumo.

El equilibrio del comercio implica que el valor de la producción es igual al valor del consumo, es decir,

$$
p^{\prime} Q=p^{\prime} C=s p^{\prime} Q_{w}
$$

donde $p$ es el vector de precios. Por lo tanto, si el comercio está equilibrado, la proporción de consumo es la proporción de producción de la producción mundial:

$$
s=p^{\prime} Q / p^{\prime} Q_{w^{\prime}}
$$

El vector de exportaciones netas es la diferencia entre la producción y el consumo:

$$
\begin{aligned}
T & =X-C \\
& =A^{-1} V-S A^{-1} V_{w} \\
& =A^{-1}\left(V-s V_{w}\right),
\end{aligned}
$$

que es $A^{-1}$ multiplicado por el vector de exceso de dotaciones de factores. Por lo tanto, la ecuación (6) de Heckscher y Ohlin expresa el comercio en función del exceso de dotaciones $\left(V-s V_{w}\right)$. Puede interpretarse como un conjunto de relaciones entre las intensidades de factores, el comercio y las dotaciones de recursos.

La mayor parte de los estudios empíricos del modelo de $\mathrm{H}-\mathrm{O}$ utilizan medidas de dos de estas variables para deducir la tercera. Por lo tanto, en la literatura empírica pueden encontrarse tres enfoques diferentes. Los estudios del contenido de factores, el más famoso de los cuales se debe a Wassily 
Leontief (1953) ${ }^{8}$, toma medidas del comercio $T$ y de las intensidades de factores $A$ de un determinado país y deduce a partir de ellas el llamado vector de abundancia de factores $\left(V-s V_{w}\right)$, donde $V$ es el vector de recursos de factores del pais y $\left(V_{w}\right)$ es el vector de recursos de factores del mundo. Dado un conjunto de coeficientes de las cantidades de factores necesarias en cada industria, el intercambio implícito de servicios de factores por medio del comercio es la media ponderada de estas cantidades necesarias de factores, donde los pesos corresponden a la proporción de las exportaciones (importaciones) totales correspondientes a las exportaciones (importaciones) de cada industria.

Los estudios en los que se consideran varios bienes realizan regresiones de las exportaciones netas con respecto a las intensidades de factores de la mercancia de un determinado país. Se considera que los coeficientes estimados son la abundancia relativa de cada factor de producción en cuestión.

Por último, los estudios en los que se consideran varios paises utilizan medidas del comercio $T$ y medidas directas de las dotaciones $V$ y deducen implicitamente la inversa de la matriz de intensidades $A$. Como señala Leamer, este tipo de estudio, aunque es conceptualmente correcto, no puede decirse que sea una medición completa de la exactitud de la teoría, ya que no utiliza ningún dato sobre las intensidades de factores. Puede decirse que el enfoque mide la exactitud de una versión más débil de la teoría que no depende de las intensidades de factores, a saber, "existe una matriz $A$ tal que $A T=V-s V_{w}$ " (Leamer, 1984, p. 59). Este es el modelo que utilizamos en este capítulo.

\section{EL TEOREMA DE HECKSCHER Y OHLIN Y LA HISTORIA ECONOMICA}

Sorprendentemente, el modelo de Heckscher y Ohlin ha sido casi olvidado por los historiadores económicos 9 . Los dos trabajos más recientes e influyentes que analizan los determinantes de la ventaja comparativa en la industria manufacturera del Reino Unido y Estados Unidos antes de la Primera Guerra Mundial y que utilizan un modelo de Heckscher y Ohlin siguen los dos prime-

${ }^{8}$ Leontief obtuvo su "paradoja» clásica cuando observó que la relación capital-trabajo que implicaban las exportaciones americanas era menor que la que implicaban las sustituciones de importaciones. El debate que provocaron los resultados de Leontief ha sido examinado por Deardorff (1984).

9 Las excepciones más destacadas son, por supuesto, las aportaciones de los propios Heckscher y Ohlin (véase Flam y Flanders, 1991). 
ros enfoques Crafts-Thomas y Wright ${ }^{10}$. Crafts y Thomas realizan una regresion considerando varias mercancias para explicar la pauta de comercio de la industria manufacturera británica entre 1910 y 1935 y comparan los resultados con los de Estados Unidos correspondientes a 1909. En concreto, estiman regresiones de las exportaciones netas con respecto a medidas del stock de capital, el capital humano y el trabajo no cualificado de cada industria. Observan que Gran Bretaña exportaba productos intensivos en capital y trabajo no cualificado y bienes importados intensivos en capital humano. Los resultados correspondientes a Estados Unidos son en sus propias palabras los siguientes: «Parece que en 1909 Estados Unidos ya sigue la pauta característica de los paises avanzados de exportar bienes intensivos en capital humano e importar bienes intensivos en trabajo no cualificado», Crafts y Thomas ${ }^{11}$.

Gavin Wright ${ }^{12}$ ha estudiado el caso de Estados Unidos con una base de datos más rica utilizando métodos input-output tradicionales y regresiones en las que se consideran varias mercancías. $\mathrm{El}$ análisis se refiere a seis años que sirven de punto de referencia: $1879,1899,1909,1914$ y 1940 . Llega a la siguiente conclusión: «Desde 1879 hasta 1928, las exportaciones americanas de bienes manufacturados fueron más intensivas en capital que las importaciones americanas», Wright ${ }^{13}$, pero «las mayores diferencias en cuanto a la intensidad de factores se encontraban en los recursos naturales no reproducibles» $y$ «no sólo las exportaciones americanas tenian un mayor contenido de recursos naturales que las importaciones, sino que esta tendencia estaba aumentando tanto en términos absolutos como en términos relativos precisamente en el periodo histórico en que el pais estaba comenzando a mostrar su superioridad mundial en la industria», Wright ${ }^{14}$. Por otra parte, realiza el contraste utilizando una regresion de mercancias que incluye un coeficiente de recursos naturales y llega a la conclusión de que «el capital y los recursos naturales eran factores de producción complementarios», Wright.

Hasta donde yo sé, nadie ha contrastado la proposición de $\mathrm{H}-\mathrm{O}$ en el caso de los años anteriores a la Primera Guerra Mundial utilizando el tercer enfoque, a saber, un análisis internacional. La metodología intersectorial, como la de Crafts y Thomas y Wright, centra la atención en un determinado pais con el fin de analizar los factores que configuraron la ventaja comparativa a lo largo del tiempo. Los problemas de este enfoque se hallan en que sólo utiliza una

\footnotetext{
10 Crafts y Thomas (1986), Wright (1990).

11 Crafts y Thomas (1986), p. 637.

12 Gavin Wright (1990).

13 Wright (1990), p. 657

14 Wright (1990), p. 658.
} 
observación (pais) para analizar los determinantes del comercio. Por otra parte, el hecho de que las variables explicativas sean las características de los factores limita su interpretación de la política (por ejemplo, si el gobierno fomentó la adopción de medidas tendentes a aumentar la tasa de formación de capital o a estimular la acumulación de capital humano). Por otra parte, como demuestran Leamer y Bowen ${ }^{15}$, hay razones para preguntarse si los signos de los coeficientes que se obtienen en estas regresiones intersectoriales del comercio con respecto a los factores del sector pueden utilizarse para deducir la influencia de los cambios de la abundancia de recursos de un país en la estructura de su comercio, a menos que cada factor sea específico de cada sector ${ }^{16}$. El análisis internacional, en comparación con el intersectorial, exige un mayor esfuerzo en la recogida de datos y, lo que es más importante, el hecho de que las estimaciones obtenidas con el primero se refieren a una determinada mercancía, mientras que las estimaciones que se obtienen con el segundo se refieran al comercio de un determinado pais, explica por qué las regresiones intersectoriales se utilizan más a menudo en la historia económica para estudiar los determinantes del comercio de un pais específico. Sin embargo, como sostiene Bowen ${ }^{17}$, el hecho de que las regresiones internacionales revelen información únicamente sobre una determinada mercancía no limita su utilización para identificar la dotación de recursos que determina la pauta de comercio de un determinado país. En el artículo antes mencionado, Bowen utiliza con éxito este tipo de análisis para explicar la evolución de la ventaja comparativa de Estados Unidos en el periodo comprendido entre 1963 y 1975. Este tipo de ejercicio necesita unos datos sobre el comercio mucho más desagregados que los que utilizamos en este capitulo.

El primer paso de un enfoque comparativo puede observarse en el estudio de Crafts ${ }^{18}$. Crafts calcula una medida de la ventaja comparativa revelada ${ }^{19}$ de 11 países y 5 años de referencia $(1899,1913,1929,1937,1950)$. Pero, como señala el autor, «el enfoque consiste, pues, en identificar los sectores en los que

15 Leamer y Bowen (1980).

is Bowen y Sveikauskas (1992) han mostrado recientemente que aunque la teoria pone en duda el método de la regresión intersectorial, es un indicador fiable de la abundancia revelada de factores cuando se realizan las correcciones adecuadas para tener en cuenta el desequilibrio del comercio.

17 Bowen (1983)

18 Crafts (1989).

19 La medida de la ventaja comparativa revelada fue introducida por Balassa (1965) y se ha utilizado a menudo en los trabajos empiricos posteriores. Los sectores de cada país se clasifican en función de la proporción de las exportaciones mundiales totales correspondientes a esa mercancia; se dice que los sectores que tienen una cuota de mercado mayor que la cuota de mercado mundial total tienen una «ventaja comparativa revelada». 
una economia tiene una ventaja comparativa más que en indicar la fuente subyacente de la ventaja comparativa en lo que se refiere a las dotaciones de factores, la tecnología, La demanda o cualquier otra cosa» 20.

K. O'Rourke y J. G. Williamson ${ }^{21}$ también han examinado el lado de la proposición relacionado con los precios, analizando la influencia de la convergencia de los precios de los bienes en la convergencia de los precios de los factores a finales del siglo xix. Observan que la mitad de la convergencia de los salarios reales de los trabajadores no cualificados de Gran Bretaña y Estados Unidos durante el periodo 1870-1913 puede atribuirse a la convergencia de los precios de las mercancias. Sus resultados son coherentes con las afirmaciones teóricas de Heckscher y Ohlin, que escribieron sus trabajos justo después de la Primera Guerra Mundial. K. O'Rourke, A. Taylor y J. G. Williamson ${ }^{22}$ también observan lo mismo en una muestra mayor de finales del siglo XIX con la que pretenden abordar los determinantes de la convergencia de los cocientes entre los salarios y los precios de alquiler del capital.

\section{EL MODELO DEL COMERCIO DE HECKSCHER Y OHLIN APLICADO A 1913}

En este apartado realizamos un contraste del paradigma de $\mathrm{H}-\mathrm{O}$ con datos de 1913. En la literatura de historia económica se utiliza a menudo 1913 de común acuerdo como año representativo del periodo anterior a 1914. El año 1913 supone el final de cuarenta años de «normalidad», tal como la describe P. L. Yates ${ }^{23}$ en su estudio clásico del comercio exterior del periodo 1913. 1953. Aunque el periodo comprendido entre la década de 1870 y la Primera Guerra Mundial se caracteriza por un enorme aumento de la población, de la producción y del comercio mundiales, todo esto ocurrió en una situación en la que los precios interiores, los tipos de cambio y las fronteras políticas eran relativamente estables. Llegamos a la conclusión de que el modelo de $\mathrm{H}-\mathrm{O}$ basado en la competencia perfecta es un paradigma totalmente adecuado del comercio de finales del siglo XIX, periodo que motivó a Eli F. Heckscher y Bertil Ohlin.

La ecuación (6) de Heckscher y Ohlin expresa el comercio en función del exceso de dotaciones $\left(V-s V_{w}\right)$. También podemos realizar una regresión con

\footnotetext{
20 Crafts (1989), p. 128.

${ }^{21}$ Kevin O'Rourke y Jeffrey G. Williamson (1992) y J. G. Williamson (1992).

22 K. O'Rourke, A. Taylor y J. G. Williamson (1993).

23 P. L. Yates (1959).
} 
fines empíricos únicamente con respecto a las dotaciones de factores. Como muestra Leamer ${ }^{24}$ (1984, p. 159), esta formulación alternativa del modelo se prefiere por razones econométricas ${ }^{25}$. Por lo tanto, estimamos la siguiente ecuación para cada agregado del comercio:

$$
N X_{i j}=a_{0 j}+a_{1 j} C A P_{j}+a_{2 j} S K I_{j}+a_{3 j} U_{N S K_{j}}+a_{4 j} A G R_{j}+a_{5 j} M I N,
$$

donde

$N X_{i j}: \quad$ exportaciones netas del grupo de bienes $i$, pais $j$

$C A P_{j}: \quad$ medida del stock total de capital, pais $j$

$S K I$ : $\quad$ población activa cualificada, país $j$

$U N S K_{j}$ : población activa no cualificada, pais $j$

$A G R_{j}: \quad$ tierra agrícola, pais $j$

$M I N_{j}: \quad$ recursos minerales, pais $j$.

La ecuación (7) se estima con datos procedentes de los dieciocho países enumerados en el cuadro 1.2a y de cada uno de los seis agregados del comercio enumerados en el cuadro $1.2 \mathrm{~b}$. Los seis grupos de mercancias son los productos agrícolas (AGPRO), las materias primas (RAWMAT), los bienes intensi-

24 Leamer (1984), p. 159.

25 El modelo de comercio de Heckscher, Ohlin y Vanek, tal como se presenta en el apartado II, puede expresar el comercio en función de las dotaciones o en función de los excesos de dotaciones. En una versión $2 \times 2$, las ecuaciones del modelo son:

$$
\begin{gathered}
T_{1}=\beta_{1 L}\left(L-Y L_{w} / Y_{w}\right)+\beta_{1 K}\left(K-Y K_{w} / Y_{w}\right) \\
T_{2}=\beta_{2 L}\left(L-Y L_{w} / Y_{w}\right)+\beta_{2 K}\left(K-Y K_{w} / Y_{w}\right) \\
Y=w_{L} L+w_{K} K,
\end{gathered}
$$

donde $T_{1}$ y $T_{2}$ son las exportaciones netas de las dos mercancias, $Y$ es el PNB, $L$ es el trabajo, $K$ es el capital, $w_{L}$ y $w_{K}$ son los rendimientos de los factores, los subindices $w$ se refieren al mundo $y$ las $\beta$ son los coeficientes de Rybczynski. Este tipo de modelo expresa el comercio neto como una función lineal del exceso de dotaciones de factores. Sin embargo, como cada uno de los excesos de dotaciones de factores es una función lineal de todas las dotaciones de factores (es decir, $\left.L-Y L_{w} / Y_{w}=L-\left(w_{L}+w_{K} K\right) L_{w} / Y_{w}\right)$ en casi todas las distribuciones de $K$ y $L$ estos excesos de dotaciones están correlacionados y una regresión del comercio con respecto a los excesos de dotaciones genera estimaciones sesgadas e incoherentes. Este problema se agrava si hay errores de medición. Como consecuencia de estos problemas, en los estudios empíricos se refiere una forma reducida del modelo. Esta forma reducida se obtiene insertando la ecuación del PNB en las ecuaciones de exportaciones netas:

$$
\begin{gathered}
T_{1}=\boldsymbol{\Theta}_{1 L} L+\boldsymbol{\Theta}_{1 K} K \\
T_{2}=\boldsymbol{\Theta}_{2 L} L+\boldsymbol{\Theta}_{2 K} K \\
Y=w_{L} L+w_{K} K .
\end{gathered}
$$




\section{CUADRO 1.2}

Muestra de paises y agregados comerciales 1913

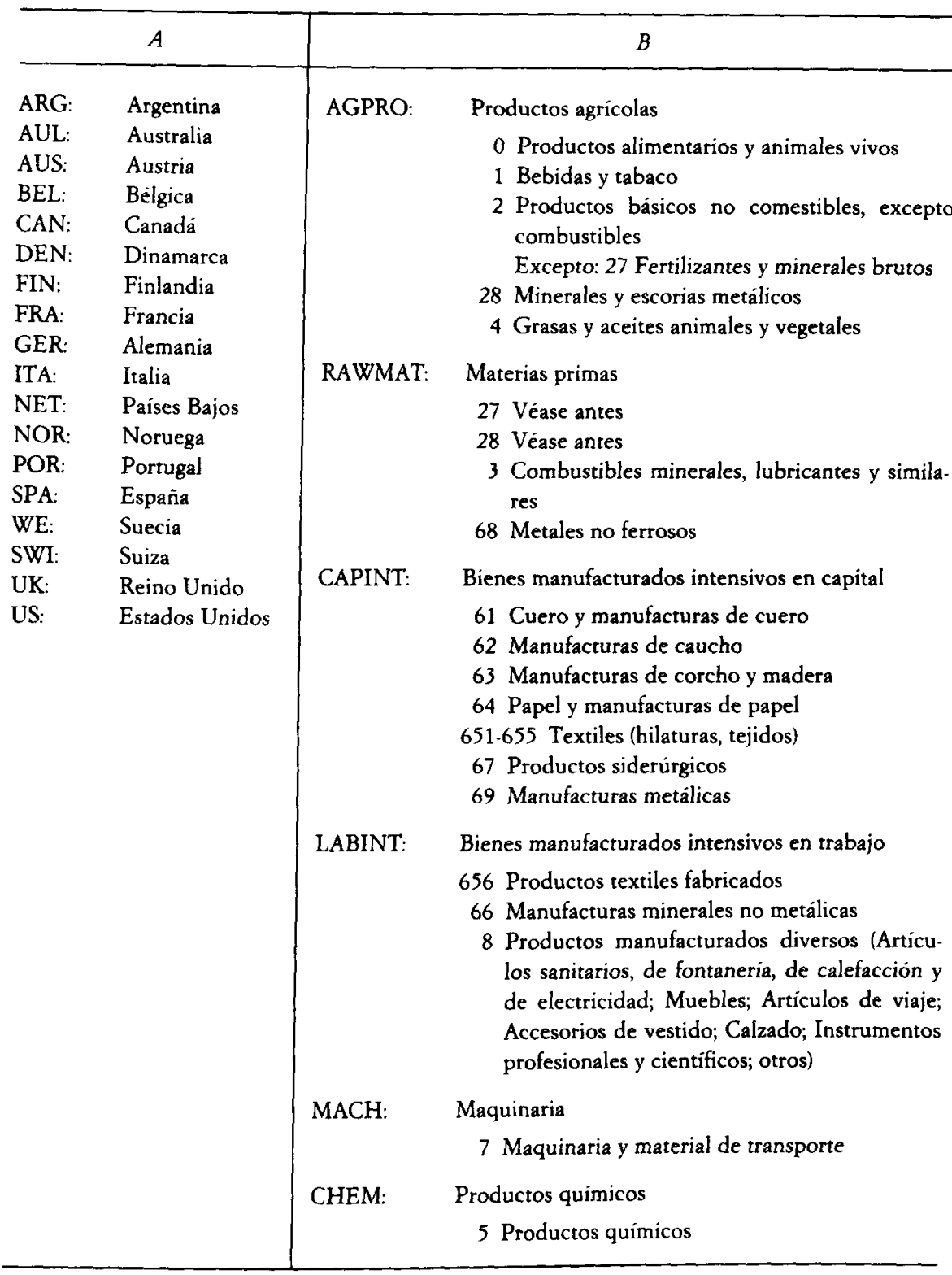

Notas: Véase el apéndice. 
vos en capital (CAPINT), los bienes intensivos en trabajo (LABINT), la maquinaria $(\mathrm{MACH})$ y los productos químicos $(\mathrm{CHEM})$. El cuadro $1.2 \mathrm{~b}$ contiene una descripción detallada y el apéndice analiza los métodos agregados utilizados para la formación de estos agregados. Las cinco dotaciones de factores utilizadas en esta estimación son una medida del stock de capital (CAP), el trabajo agrupado por cualificaciones utilizando una variable del nivel de estudios (SKI y UNSK), la tierra agrícola (AGR) y los recursos minerales (MIN). Salvo en el caso de las variables de la tierra y la población activa, el resto de estas variables explicativas se ha elaborado a partir de fuentes primarias. El análisis de las fuentes y de los métodos utilizados se encuentra en el apéndice.

Dado que no tenemos medidas comparables del stock de capital (CAP) de esta gran muestra de países, utilizamos como variable aproximada el consumo de combustibles sólidos en $1913^{26}$. Los datos sobre el consumo de energía se refieren al consumo aparente de fuentes primarias, incluidas las importaciones netas de tipos de energía secundarios y primarios. Debido a los datos existentes, sólo se han tenido en cuenta los combustibles sólidos (carbón de antracita, lignito y coque). Para poder hacer agregaciones y comparaciones, los datos se expresan en miles de unidades equivalentes de carbón de antracita ${ }^{27}$. El consumo de combustibles sólidos fue, en general, la fuente más importante de energía hasta la Primera Guerra Mundial. Incluso en los paises de la periferia europea pobres en carbón y en los que la utilización de madera como combustible (especialmente los países escandinavos) o energía hidroeléctrica (en particular, Italia y Suiza) era una importante fuente de energía, la correlación entre el consumo de carbón y los niveles de industrialización es muy alta ${ }^{28}$.

26 Sólo se dispone del stock de capital fijo no residencial existente en 1913 en el caso de unos cuantos paises: Francia, Alemania, Italia, Japón, Reino Unido y Estados Unidos (véase Maddison, 1982). Otra medida considerada es la cantidad efectiva de caballos de vapor (maquinaria, ferrocarriles y barcos de vapor). Existen algunas estimaciones aproximadas correspondientes a 1895 (véase Mulhall, 1896, 1989, y Woytinsky, 1926), aunque sólo se refieren a 13 paises (Australia, Austria, Bélgica, Canadá, Dinamarca, Francia, Alemania, Italia, Países Bajos, España, Suiza, Reino Unido y Estados Unidos).

27 Véase el apéndice para una descripción de esta medida.

${ }_{28}$ El problema más inmediato se deriva de la utilización de una variable flujo como una variable aproximada de un stock. No se ha intentado en este articulo utilizar este enfoque. Sin embargo, la utilización del consumo de energia se ha empleado a menudo como variable aproximada del capital. Véase a título de ejemplo el trabajo clásico de Landes (1969), quien, citando el de A. G. Frank (1959), afirma lo siguiente: «El coeficiente de correlación entre el consumo de energía y los cálculos que se han hecho del stock de capital industrial es asombrosamente alto: en el caso de Estados Unidos, 0,9995 en el periodo 1880-1948; en el del Reino Unido, 0,96 ó 0,99 en el periodo 1865-1914, dependiendo de la serie empleada. De hecho, uno casi se siente tentado a preguntarse si merece la pena el esfuerzo de elaborar la medición compuesta directa de la formación de capital» (p. 293). En un reciente estudio, P. Fraile (1992) muestra en el caso de las econo- 
La dotación de trabajo se divide en trabajo no cualificado, UNSK (la población activa multiplicada por la tasa de analfabetismo) y trabajo cualificado, SKI (la población activa menos el trabajo no cualificado). Los datos se expresan en miles de trabajadores. La tasa de analfabetismo es el porcentaje de la población de 10 años o más que no sabe leer y escribir. En este artículo, la alfabetización se utiliza como medida objetiva del nivel de estudios. La escolarización elemental (los tipos más avanzados de educación constituyen una medida menos fiable para realizar comparaciones internacionales) es otra medida. Sin embargo, los años de escolarización son un estimador menos preciso del nivel de estudios elementales que los niveles de alfabetización, principalmente por dos razones. En primer lugar, la eficacia de la enseñanza puede variar de unos países a otros. En segundo lugar, en las primeras etapas del desarrollo, muchas personas aprenden a leer y a escribir fuera de las instituciones educativas formales. Por último, en lugar de medir los resultados del sector educativo por los factores que utiliza, las tasas de alfabetización nos permiten juzgarlo por su producción (Tortella, 1990). A causa de la falta de datos, no se ha intentado ajustar estas variables para tener en cuenta las tasas de analfabetismo femenino y las tasas de actividad femeninas.

La dotación de tiera es la tierra agrícola (AGR) y procede del estudio de la Sociedad de Naciones ${ }^{29}$ sobre $1913^{30}$. Los datos se expresan en miles de hectáreas. También se han utilizado otras medidas de la tierra para estimar la ecuación (7) y se han obtenido resultados similares. En concreto, la tierra productiva y la tierra arable indicadas por la Sociedad de Naciones ofrecen resultados similares. El área total dividida en zonas climáticas, como hace Leamer, también es coherente con el modelo ${ }^{31}$.

Por último, la variable de la dotación de minerales (MIN) es una variable que mide de forma aproximada la dotación de recursos naturales por medio de la producción interior de petróleo y de un compuesto de minerales: bauxita, cobre, mineral de hierro, plomo, manganeso, níquel, fosfato, potasio, piritas,

mias más periféricas correlaciones superiores a 0,95 entre los indices de producción industrial y el consumo de carbón entre 1880 y 1913 en el caso de Austria, Italia, Rusia, Suecia y España. Además, partiendo de datos de 1958, Leamer (1984) calcula que la correlación entre el consumo de energia (incluidos los combustibles sólidos, los combustibles líquidos, el gas natural y la energia hidroeléctrica) y el stock de capital medido como los flujos de inversión bruta acumulada y descontada registrados desde 1948, suponiendo que la vida media es de 15 años, es muy alta: 0,98.

${ }^{29}$ Sociedad de Naciones (1927b).

30 La tierra agricola se define en el estudio de la Sociedad de Naciones (1927b) de la manera siguiente: «tierra arable, pastos, praderas, árboles, arbustos y matorrales, pero no bosques». Los datos de los países considerados son bastante comparables.

${ }^{31}$ Leamer (1984). 
azufre, estaño y zinc. Para agregar estas variables se han utilizado los precios de los mercados internacionales.

Los cuadros 1.3 (A) y 1.3 (B) contienen los resultados de las regresiones realizadas utilizando el método de los mínimos cuadrados ordinarios. Se han realizado contrastes de la heterocedasticidad, partiendo de la hipótesis de que estaba relacionada con la existencia de capital o con el PNB total. Los resultados no confirman la presencia de heterocedasticidad relacionada con estas variables. Sin embargo, como se trata de un ejercicio de corte transversal, el cuadro 1.3 (A) muestra los errores típicos calculados de acuerdo con el procedimiento introducido por Hal White (1980) para tener en cuenta la heterocedasticidad desconocida en la estructura de los errores ${ }^{32}$. En el cuadro 1.3 (B) se muestran los resultados de las regresiones realizadas utilizando el método de los mínimos cuadrados ordinarios habituales. Las $\mathrm{R}^{2}$ normalmente son muy altas y la mayoria de los coeficientes estimados tienen el signo correcto y son estadísticamente significativos. Los resultados se mantienen cuando se altera la manera en que se definen las variables. En concreto, la utilización del número de personas matriculadas en estudios secundarios como medida de la cualificación o la introducción de diferentes tipos de tierra no alteran los resultados.

Los coeficientes mostrados en el cuadro 1.3 (A) dependen de las unidades en que se midan las variables explicativas. Como no sólo nos interesa la significación estadística de un coeficiente sino también saber hasta qué punto son importantes cada una de las variables en la explicación de la pauta de comercio, el cuadro 1.4 muestra los valores de los coeficientes beta beta de cada una de las cinco variables explicativas correspondientes a cada agregado del comercio considerado. Es posible que un coeficiente adopte un valor tan pequeño que no sea significativo en ningún sentido, salvo el estadístico. Un coeficiente beta es igual al coeficiente estimado multiplicado por el cociente entre la desviación típica de la variable explicativa y el error típico de la variable dependiente ${ }^{33}$. Estos valores beta son directamente proporcionales a la contribución de cada variable a la predicción del comercio neto. Estos valores indican cuánto cambia la variable del comercio neto, expresada en unidades de desviación típica cuando varia la dotación de factores en una desviación típica. Un valor beta de 0,1 es bajo, ya que una variación del recurso en una desviación típica apenas produciria un efecto perceptible en las exportaciones netas, pero

32 Se ha utilizado la opción ROBUST del paquete estadístico TSP (versión 4.2) para obtener las estimaciones coherentes que tienen en cuenta la heterocedasticidad siguiendo el procedimiento de Hal White (1980).

${ }^{3}$ Maddala (1977), Leamer (1988). 
un valor de uno puede considerarse alto. Si suponemos arbitrariamente, como hace Leamer ${ }^{34}$, que 1,0 define un valor beta significativo, CAP es significativo 5 veces, AGR 4 veces y SKI y MIN 3 veces.

\section{CUADRO 1.3 (A)}

El modelo de Heckscher, Oblin y Vanek en 1913 (estimaciones por MCO, consistentes para tener en cuenta la heterocedasticidad)

\begin{tabular}{|c|c|c|c|c|c|c|c|}
\hline & $C A P$ & $S K I$ & UNSK & $A G R$ & $M I N$ & $R 2$ & $\operatorname{Adj} \mathrm{R} 2$ \\
\hline AGPRO & $\begin{array}{c}-7,6 * * * \\
(5,22)\end{array}$ & $\begin{array}{l}-26,3 \\
(1,74)\end{array}$ & $\begin{array}{r}-31,9 \\
(0,55)\end{array}$ & $\begin{array}{l}8,5^{\star \star} \\
(2,38)\end{array}$ & $\begin{array}{l}4,5 * * * \\
(4,50)\end{array}$ & 0,81 & 0,73 \\
\hline RAWMAT & $\begin{array}{l}2,7^{* * *} \\
(5,22)\end{array}$ & $\begin{array}{c}-20,6 \star \star \star * \\
(4,61)\end{array}$ & $\begin{array}{l}41,6 \text { * } \\
(2,14)\end{array}$ & $\begin{array}{c}-3,0 * * * \\
(4,91)\end{array}$ & $\begin{array}{l}0,4 \\
(1,67)\end{array}$ & 0,78 & 0,69 \\
\hline CAPINT & $\begin{array}{l}2,1 * * * \\
(5,98)\end{array}$ & $\begin{array}{l}18,4 * * * \\
(4,21)\end{array}$ & $\begin{array}{c}13,3 \\
(0,66)\end{array}$ & $\begin{array}{c}-6,5 * * * \\
(4,98)\end{array}$ & $\begin{array}{r}-0,7 * \\
(2,08)\end{array}$ & 0,83 & 0,77 \\
\hline LABINT ....... & $\begin{array}{c}-0,9 * * * \\
(3,20)\end{array}$ & $\begin{array}{l}17,8^{* * *} \\
(8,89)\end{array}$ & $\begin{array}{l}-9,8 \\
(1,24)\end{array}$ & 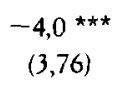 & $\begin{array}{l}0,8 \text { ** } \\
(2,59)\end{array}$ & 0,65 & 0,51 \\
\hline $\mathrm{MACH}$. & $\begin{array}{l}1,1 * * * \\
(4,73)\end{array}$ & $\begin{array}{r}5,0 * \\
(2,12)\end{array}$ & $\begin{array}{l}-9,6 \\
(1,05)\end{array}$ & $\begin{array}{c}-3,1 * * * \\
(5,87)\end{array}$ & $\begin{array}{l}0,08 \\
(0,54)\end{array}$ & 0,91 & 0,88 \\
\hline CHEM ......... & $\begin{array}{l}-0,1 \\
(1,08)\end{array}$ & $\begin{array}{l}6,7^{* * *} \\
(5,87)\end{array}$ & $\begin{array}{l}-6,3 \\
(1,62)\end{array}$ & $\begin{array}{c}-2,3 * \star \\
(2,53)\end{array}$ & $\begin{array}{l}0,38 \\
(1,72)\end{array}$ & 0,69 & 0,56 \\
\hline
\end{tabular}

NOTAS: Estadisticos $t$ (entre paréntesis) ajustados para tener en cuenta la heterocedasticidad siguiendo el procedimiento de White (1980). Los valores criticos (contraste de dos colas) proceden de la distribución de la $t$ de Student con 12 grados de libertad.

* significativo al nivel de $10 \%$.

** significativo al nivel de $5 \%$.

*** significativo al nivel de $1 \%$.

FUENTE: Véase el apéndice.

En términos generales, utilizando las estimaciones de los cuadros 1.3 (A) y 1.3 (B), la ventaja comparativa de los productos agrícolas (AGPRO) va unida a la abundancia de tierra y recursos minerales y está relacionada negativamente con el capital. El comercio de RAWMAT debe su ventaja comparativa a la existencia de capital y trabajo no cualificado. La tierra y el trabajo no cualificado contribuyen a la desventaja comparativa. Las fuentes de la ventaja compa-

34 Leamer (1984), p. 177. 
rativa en la industria manufacturera son, en general, las esperadas: el capital es una fuente de ventaja comparativa en el caso de los bienes CAPINT y MACH. Los recursos minerales son importantes en los grupos LABINT y CHEM. El trabajo cualificado contribuye también a la ventaja comparativa en todos los grupos de bienes manufacturados. Los valores beta indican de nuevo que la contribución es más importante en LABINT y CHEM, seguidos de CAPINT y $\mathrm{MACH}$. Las exportaciones netas de todos los grupos de bienes manufacturados están relacionadas negativamente con la oferta de tierra ${ }^{35}$.

\section{CUADRO 1.3 (B)}

El modelo de Heckscher, Oblin y Vanek en 1913 (estimaciones por MCO, ecuación 7)

\begin{tabular}{|c|c|c|c|c|c|c|c|}
\hline & $C A P$ & $S K I$ & UNSK & $A G R$ & MIN & $R 2$ & Adj R2 \\
\hline AGPRO $\ldots . .$. & $\begin{array}{c}-7,6 * * * \\
(3,14)\end{array}$ & $\begin{array}{c}-26,3 \\
(1,08)\end{array}$ & $\begin{array}{r}-31,9 \\
(0,54)\end{array}$ & $\begin{array}{l}8,5 \\
(1,66)\end{array}$ & $\begin{array}{l}4,5 * * \pi \\
(3,54)\end{array}$ & 0,81 & 0,73 \\
\hline RAWMAT. & $\begin{array}{l}2,7 * * * \\
(3,77)\end{array}$ & $\begin{array}{c}-20,6 * * \\
(2,87)\end{array}$ & $\begin{array}{l}41,6 \\
(2,42)\end{array}$ & $\begin{array}{r}-3,0^{*} \\
(2,04)\end{array}$ & $\begin{array}{l}0,4 \\
(1,06)\end{array}$ & 0,78 & 0,69 \\
\hline CAPINT & $\begin{array}{l}2,1 \text { ** } \\
(2,44)\end{array}$ & $\begin{array}{l}18,4 \text { * } \\
(2,11)\end{array}$ & $\begin{array}{l}13,3 \\
(0,63)\end{array}$ & $\begin{array}{c}-6,5^{\star * *} \\
(3,52)\end{array}$ & $\begin{array}{l}-0,7 \\
\quad(1,56)\end{array}$ & 0,83 & 0,77 \\
\hline LABINT & $\begin{array}{c}-0,9 \\
(1,53)\end{array}$ & $\begin{array}{l}17,8^{\star \star} \\
(2,77)\end{array}$ & $\begin{array}{l}-9,8 \\
(0,63)\end{array}$ & $\begin{array}{c}-4,0 \star \star \\
(2,99)\end{array}$ & $\begin{array}{l}0,8 * \star \\
(2,54)\end{array}$ & 0,65 & 0,51 \\
\hline MACH $\ldots \ldots \ldots$ & $\begin{array}{l}1,1^{* \star *} \\
(3,40)\end{array}$ & $\begin{array}{c}5,0 \\
(1,46)\end{array}$ & $\begin{array}{l}-9,6 \\
(1,15)\end{array}$ & $\begin{array}{c}-3,1 * * * \\
(4,30)\end{array}$ & $\begin{array}{l}0,08 \\
(0,46)\end{array}$ & 0,91 & 0,88 \\
\hline CHEM . & $\begin{array}{c}-0,1 \\
(0,48)\end{array}$ & $\begin{array}{c}6,7^{*} \\
(2,16)\end{array}$ & $\begin{array}{l}-6,3 \\
(0,83)\end{array}$ & $\begin{array}{c}-2,3 * * * \\
(3,50)\end{array}$ & $\begin{array}{l}0,38 \text { ** } \\
(2,31)\end{array}$ & 0,69 & 0,56 \\
\hline
\end{tabular}

Notas: Estadísticos $t$ (entre paréntesis) no ajustados para tener en cuenta la heterocedasticidad siguiendo el procedimiento de White (1980). Los valores críticos (contraste dos colas) proceden de la distribución de la $t$ de Student con 12 grados de libertad.

* significativo al nivel de $10 \%$.

** significativo al nivel de $5 \%$.

$\star \star \star$ significativo al nivel de $1 \%$.

FuENTE: Véase el apéndice.

35 No deberian sorprendernos algunos resultados como que la tierra agricola influye negativamente en la ventaja comparativa de todos los grupos de bienes manufacturados. Aunque el modelo utilizado aqui y resumido en la ecuación $Q=A^{-1} V$ parece exigir que todos los factores se utilicen en todas las industrias, no es asi. La existencia de factores especificos de determinados 
CUADRO 1.4

El modelo de Heckscher, Oblin y Vanek en 1913

(coeficientes beta, ecuación 7)

\begin{tabular}{|c|c|c|c|c|c|}
\hline & $C A P$ & $S K I$ & UNSK & $A G R$ & $M I N$ \\
\hline AGPRO .. & $-1,83$ & $-0,51$ & $-0,11$ & 0,72 & 1,64 \\
\hline RAWMAT. & 2,36 & $-1,45$ & 0,50 & $-0,92$ & 0,53 \\
\hline CAPINT ...... & 1,30 & 0,92 & 0,11 & $-1,42$ & $-0,65$ \\
\hline LABINT ..... & $-1,10$ & 1,76 & $-0,17$ & $-1,73$ & 1,48 \\
\hline 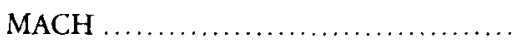 & 1,22 & 0,45 & $-0,15$ & $-1,22$ & 0,13 \\
\hline 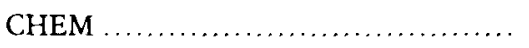 & $-0,24$ & 1,29 & $-0,21$ & $-1,93$ & 1,37 \\
\hline
\end{tabular}

FUENTE: Véase el apéndice.

Para contrastar la solidez de estas estimaciones se ha realizado un análisis de sensibilidad. Se han identificado las observaciones influyentes utilizando los estadísticos $t$ extremos de las variables ficticias que seleccionan un único país y que se incluyen de una en una en la ecuación (cuadro 1.5) ${ }^{36}$. Un valor positivo (negativo) identifica el país como una observación extrema o influyente en el sentido de que tiene unas grandes (bajas) exportaciones netas en relación con el resto de la muestra. Sin embargo, en general, los coeficientes de los cuadros 1.3 (A) y (B) que tienen unos estadísticos $t$ altos son insensibles a la omisión de esas observaciones. En los dos apartados siguientes ofrecemos un análisis más detallado de esta conducta atípica.

sectores implica que algunos elementos de la matriz de cantidades necesarias de factores $A$ puede ser cero. Por ejemplo, en un modelo de dos factores, trabajo $L$ y tierra $M$, y dos bienes, bienes agricolas $X_{1}$ y bienes industriales $X_{2}$, si la tierra no se utiliza para producir el bien industrial, el elemento $a_{M 2}$ de la matriz $A$ será cero. Puede demostrarse fácilmente que incluso aunque para producir el bien agricola se utilice tanto trabajo como tierra, la producción de bienes agrícolas sólo depende de la dotación de tierra. Y aunque la tierra no se utilice para producir bienes in. dustriales, el nivel de producción de bienes industriales depende tanto de la dotación de trabajo como de la dotación de tierra. Este resultado aparentemente paradójico se debe al hecho de que el pleno empleo exige que la tierra se utilice totalmente en el sector agrícola. Este hecho, junto con la cantidad fija necesaria de factores $a_{M 1}$, determina el nivel de producción agricola $M / a_{M 1}$. Dado que el residuo de trabajo que queda para la producción industrial depende, pues, de la dotación de tierra (es decir, $L-X_{1} a_{L 1}=L-M a_{L 1} / a_{M 1}$ ), resulta evidente que el nivel de producción industrial tambièn depende de la dotación de tierra.

36 Las variables ficticias de los paises se han incluido en la ecuación (7) de una en una. Se ha utilizado el método de estimación de mínimos cuadrados ordinarios ajustado para tener en cuenta la heterocedasticidad utilizando el procedimiento de White (1980). 


\section{CUADRO 1.5}

El modelo de Heckscher, Oblin y Vanek en 1913 (estadísticos $t$ de las variables ficticias de los países, ecuación 7 )

\begin{tabular}{|c|c|c|c|c|c|c|}
\hline & $A G P R O$ & $R A W M A T$ & CAPINT & $L A B I N T$ & $M A C H$ & CHEM \\
\hline Argentina $\ldots \ldots \ldots \ldots$ & 1,8 * & 0,3 & $-0,3$ & 0,2 & 0,1 & 0,1 \\
\hline Australia $\ldots \ldots \ldots \ldots \ldots$ & $-0,4$ & 0,5 & $-1,0$ & $-2,3 * *$ & $-1,0$ & $-1,4$ \\
\hline Austria $\ldots \ldots \ldots \ldots \ldots$ & 0,0 & $-0,5$ & $-0,3$ & $-0,6$ & 0.6 & 0,6 \\
\hline Bélgica $\ldots . . . \ldots \ldots \ldots \ldots$ & 0,1 & $-0,8$ & $-0,1$ & 0,0 & $-0,6$ & $-0,3$ \\
\hline Canadá ..... & 0,2 & $-0,2$ & 0,9 & 0,0 & 0,8 & 1,7 * \\
\hline Dinamarca $\ldots \ldots \ldots \ldots$ & 0,7 & 0,2 & $-0,4$ & $-0,2$ & $-0,0$ & 0,0 \\
\hline Finlandia $\ldots \ldots \ldots \ldots \ldots$ & 0,4 & 0,1 & $-0,1$ & 0,0 & 0,2 & 0,1 \\
\hline Francia $\ldots \ldots \ldots \ldots \ldots$ & $-1,1$ & 0,9 & 0,3 & 0,3 & $-1,3$ & $-0,1$ \\
\hline Alemania............ & 1,2 & 0,0 & 1,3 & $2,1 *$ & 1,4 & $6,1^{* * *}$ \\
\hline Italia $\ldots \ldots \ldots \ldots \ldots$ & 1,9 * & $-2,6 \star \star \star$ & $-1,6$ & $-0,7$ & $2,5 * *$ & 0,1 \\
\hline Paises Bajos ............ & 0,2 & $-0,2$ & $-1,5$ & $-0,4$ & $-0,7$ & $-0,4$ \\
\hline Noruega $\ldots \ldots \ldots \ldots . . . .$. & $-0,4$ & 0,1 & $-0,1$ & $-0,9$ & $-0,4$ & $-0,2$ \\
\hline Portugal $\ldots \ldots \ldots \ldots \ldots$ & 0,3 & $-0,4$ & $-0,3$ & 0,2 & 0,6 & 0,4 \\
\hline España $\ldots \ldots \ldots \ldots \ldots \ldots$ & $-3,2 * \star \star$ & $3,6 * * *$ & 2,9 ** & 0,7 & $-3,1^{\star \star \star}$ & $-0,3$ \\
\hline Suecia $\ldots \ldots \ldots \ldots \ldots$ & 0,0 & 0,6 & 0,0 & $-0,6$ & 0,1 & $-0,6$ \\
\hline Suiza $\ldots \ldots \ldots \ldots \ldots \ldots$ & $-1,6$ & $-1,9$ * & 1,8 * & 2,9 ** & 1,5 & $-1,5$ \\
\hline Reino Unido ........... & $-2,5$ ** & $4,1 * \star \star$ & 1,3 & $-1,1$ & $-3,0 * \star$ & $-1,0$ \\
\hline Estados Unidos ......... & $-0,4$ & $-1,6$ & $-2,2$ ** & $-1,0$ & 0,4 & $-2,7^{* *}$ \\
\hline
\end{tabular}

NOTAS: El valor de la $t$ se refiere a la variable ficticia obtenida seleccionando en cada ecuación un país de cada vez. Los valores críticos (contraste de dos colas) proceden de la distribución de la $t$ de Student con 12 grados de libertad.

* significativo al nivel de $10 \%$.

** significativo al nivel de $5 \%$.

*** significativo al nivel de $1 \%$.

FUENTE: Véase el apéndice.

\section{DEPENDENCIA COMERCIAL Y POLITICA COMERCIAL}

Existen varias razones por las que las exportaciones netas pueden considerarse peculiares cuando se utiliza el modelo $\mathrm{H}$-O lineal como marco explicativo ${ }^{37}$. En primer lugar, el modelo lineal depende fundamentalmente de la especialización incompleta y de los rendimientos constantes de escala. Es difícil

37 Leamer (1988). 
estudiar la presencia de no linealidades cuando se utiliza una muestra relativamente pequeña, como ocurre en este artículo. En segundo lugar, el modelo puede no tener en cuenta los recursos omitidos que influyen significativamente en el comercio de paises y bienes especificos. También deberían preocuparnos los errores de medición de algunos de los recursos. En tercer lugar, el nivel de agregación utilizado en los datos sobre el comercio es arbitrario y no existe garantía alguna de que los resultados no dependan del nivel de agregación de las mercancias elegido. Está elaborándose un conjunto más desagregado de datos para resolver estos problemas de agregación. La cuarta razón por la que las pautas de comercio pueden no ajustarse a la norma $\mathrm{H}$-O sería la presencia de barreras comerciales excepcionalmente altas o bajas, bien naturales o artificiales ${ }^{38}$. El modelo no predice la pauta de comercio en condiciones de libre comercio, sino el comercio de cada país partiendo de un nivel de protección mundial medio.

En este apartado ofrecemos algunos análisis empiricos cuyo propósito es discriminar entre diferentes explicaciones de los residuos. Las pautas de comercio que se alejan de la norma podrian atribuirse a los aranceles si los países son similares en lo que se refiere a los recursos, los gustos y las barreras naturales o si se tienen en cuenta estos efectos. Estimamos, en primer lugar, un modelo ampliado teniendo en cuenta los costes de transporte o las barreras comerciales naturales, incluyendo una nueva variable basada en la distancia con respecto a los mercados. Para averiguar si los residuos globales del modelo pueden atribuirse a las barreras comerciales, construimos un indice ajustado del comercio a partir de este modelo ampliado y vemos en qué medida está correlacionado con las medidas convencionales de la protección. En el siguiente apartado estudiamos los países que muestran una conducta atípica desde el punto de vista del modelo estimado.

Como nos interesan los residuos del modelo, hemos estimado una ecuación más general de dependencia comercial que incluye la distancia con respecto a los mercados ${ }^{39}$. Por lo tanto, la ecuación (7) se convierte en la (8):

$$
N X_{i j}=a_{0 j}+a_{1 j} C A P_{j}+a_{2 j} S K I_{j}+a_{3 j} U N S K_{j}+a_{4 j} A G R_{j}+a_{5 j} M I N_{j}+a_{6 j} D I S_{p}
$$

${ }_{38}$ Dado que las $R^{2}$ ajustadas del modelo estimado son muy altas, suponemos que no hay recursos omitidos y que los residuos podrian atribuirse a distorsiones provocadas por la política comercial. Véase el siguiente apartado para un análisis más detallado.

${ }^{39} \mathrm{La}$ introducción de esta variable independiente adicional se ha utilizado a menudo en los estudios empíricos modernos para tener en cuenta los costes de transporte o las barreras comerciales naturales. Véase, por ejemplo, Leamer (1974), Saxonhouse $(1983,1986)$ y Lawrence (1987). 
donde

$N X_{i j}: \quad$ exportaciones netas del grupo de bienes $i$, país $j$

$C A P_{j}: \quad$ medida del stock total de capital, país $j$

$S K I_{j}: \quad$ población activa cualificada, pais $j$

UNSK: población activa no cualificada, pais $j$

$A G R_{j}: \quad$ tierra agricola, país $j$

$M I N_{j}: \quad$ recursos minerales, país $j$

$D I S_{j}: \quad$ distancia entre el país $j$ y los mercados ponderada por el PNB

La distancia entra en el modelo como la distancia media en línea recta existente entre las capitales, ponderada por el PNB ${ }^{40}$. Debe interpretarse como una variable aproximada de las barreras comerciales naturales y se espera que esté relacionada negativamente con el valor absoluto de las exportaciones netas. El cuadro 1.6 muestra los resultados. Esta nueva variable sólo es significativa y tiene el signo correcto en dos categorías de bienes manufacturados: los bienes CAPINT y LABINT. Se trata de bienes manufacturados que tienen un elevado contenido de valor añadido en los que los costes de transporte son importantes en la determinación de su competitividad en los mercados extranjeros. En conjunto, el poder explicativo del modelo sólo es algo mayor, medido por los valores de las $\mathrm{R}^{2}$, en comparación con el anterior.

Siguiendo a Leamer ${ }^{41}$, hemos elaborado un indicador de la apertura basada en la estimación de este modelo $\mathrm{H}-\mathrm{O}$ ampliado. Este indicador se ha utilizado a menudo para analizar la pauta comercial de Japón ${ }^{42}$ y la orientación de los países en vías de desarrollo hacia el exterior ${ }^{43}$. Este indicador de la apertura o indice ajustado del comercio correspondiente al comercio total es la suma de las desviaciones del nivel predicho de exportaciones netas de todas las mercancías o subgrupo de mercancías con respecto al realmente observado ${ }^{44}$.

40 La variable distancia $D I S_{\text {j }}$ se pondera de la manera siguiente:

$$
D I S_{1}=\boldsymbol{\Sigma}\left(Y_{i} / D I S_{i j}\right) /(\boldsymbol{\Sigma} Y i),
$$

donde $D I S_{j i}$ es la distancia en linea recta que hay desde el capital del pais $i$ hasta el capital del pais $j$, como en Fitzpatrick (1986) e Yi es el PNB del país $i$.

41 Leamer (1988).

42 Véase, por ejemplo, Saxonhouse $(1983,1986)$ y Lawrence (1987).

43 Véase Pritchett (1991).

44 El supuesto consiste en que la mayoria de las medidas reducen los incentivos para comerciar y en que, por lo tanto, a menor intervención, mayor comercio. Sin embargo, muchas medidas de finales del siglo xix fomentaron el comercio: el nombramiento de agregados comerciales, las 
Estas desviaciones se expresan en porcentaje del PNB. Este método para detectar el proteccionismo no puede constrastarse correctamente en ausencia de medidas directas de las barreras, sobre todo durante los años posteriores a la Segunda Guerra Mundial, en que las barreras no arancelarias cobraron una creciente importancia. Sin embargo, las medidas arancelarias fueron con mucho el instrumento más importante de la política comercial hasta la Primera Guerra Mundial 45. Sin embargo, un estudio serio de este periodo también debería tener en cuenta los tratados bilaterales y la política comercial colonial ${ }^{46}$. Por otra parte, la influencia en la asignación de los recursos sólo puede valorarse con precisión por medio de medidas de la protección efectiva ${ }^{47}$. Desgraciadamente, para realizar un estudio comparativo como éste, sólo se dispone de medidas generales de la protección, a saber, los aranceles sobre las importaciones en porcentaje del total de importaciones ${ }^{48}$. En este apartado ofrecemos un enfoque provisional basado en el modelo antes estimado para medir las distorsiones provocadas por la política comercial seguida por los diferentes paises justo antes del estallido de la Primera Guerra Mundial. Para analizar exhaustivamente la influencia de los aranceles en las pautas comerciales, necesitaríamos un enfoque mucho más desagregado que el que presentamos en este articulo. Como no tenemos aún un conjunto de datos desagregados sobre las exportaciones netas y los aranceles, este análisis debería considerarse preliminar y se ampliará en nuevas investigaciones.

cámaras de comercio, las exposiciones internacionales, los almacenes de muestras para la exportación o los agentes privados. Para recoger estos efectos positivos y negativos de la política, se ha afirmado que deben utilizarse medidas alternativas de la apertura basadas en las tasas de intervención. Para un análisis, véase Leamer (1988).

45 Véase Bairoch (1989) y Pollard (1982) para un análisis de la política comercial a finales del siglo XIX.

46 Véase Bairoch (1989, pp. 103-127) y Pollard (1982).

47 Apenas se ha intentado estimar la protección efectiva existente en este importante periodo. Sólo existen estudios sectoriales de algunos países. Véase, por ejemplo, Hawke (1975) en el caso de Estados Unidos, Toniolo (1977) en el de Italia o Lains (1987) en el de Portugal.

48 El problema más importante que plantea el cálculo de los niveles arancelarios existentes en este periodo se halla en que casi todos los aranceles sobre las importaciones eran especificos. Como señala Bairoch $(1989$, p. 71), los únicos paises europeos en los que los aranceles especificos no representaban al menos el 90 por ciento de todos los aranceles sobre las importaciones eran los Países Bajos (donde la mayoría de los aranceles eran ad valorem) y Bélgica (donde una proporción significativa era ad valorem). Liepmann (1938) ofrece una medida alternativa que consiste en un «nivel arancelario potencial» de 15 paises europeos elaborada utilizando un grupo de 144 mercancías muy comerciadas. Sin embargo, Liepmann no incluye cifras de Argentina, Australia, Canadá, Noruega, Portugal, Estados Unidos, Dinamarca, Países Bajos y Reino Unido. 


\section{CUADRO 1.6}

El modelo de Heckscher, Oblin y Vanek en 1913

(estimaciones por MCO, consistentes para tener en cuenta la beterocedasticidad) (ecuación 7)

\begin{tabular}{|c|c|c|c|c|c|c|c|c|}
\hline & $C A P$ & $S K I$ & UNSK & $A G R$ & $M I N$ & DIS & $R 2$ & $\operatorname{Adj} R 2$ \\
\hline AGPRO $\ldots \ldots \ldots$ & $\begin{array}{c}-7,5 * \star \star \\
(4,62)\end{array}$ & $\begin{array}{r}-25,2 \\
(1,68)\end{array}$ & $\begin{array}{r}-29,9 \\
(0,53)\end{array}$ & $\begin{array}{l}8,4^{* *} \\
(2,29)\end{array}$ & $\begin{array}{l}4,3^{* * \star} \\
(3,67)\end{array}$ & $\begin{array}{l}88,9 \\
(0,56)\end{array}$ & 0,82 & 0,72 \\
\hline RAWMAT & $\begin{array}{l}2,7^{* \star *} \\
(5,60)\end{array}$ & $\begin{array}{c}-20,2^{\star * *} \\
(4,25)\end{array}$ & $\begin{array}{l}42,4 \text { * } \\
(2,14)\end{array}$ & $\begin{array}{c}-3,1^{* * *} \\
(5,25)\end{array}$ & $\begin{array}{l}0,3 \\
(1,41)\end{array}$ & $\begin{array}{l}32,7 \\
(1,56)\end{array}$ & 0,79 & 0.68 \\
\hline CAPINT $\ldots \ldots \ldots \ldots$ & $\begin{array}{l}2,0^{\star \star \star \star} \\
(4,76)\end{array}$ & $\begin{array}{l}17,6^{\star \star \star} \\
(4,28)\end{array}$ & $\begin{array}{c}11,7 \\
(0,63)\end{array}$ & $\begin{array}{c}-6,4^{\star \star *} \\
(6,24)\end{array}$ & $\begin{array}{r}-0,6 \text { * } \\
(2,00)\end{array}$ & $\begin{array}{c}-71,8^{\star \star} \\
(2,74)\end{array}$ & 0,85 & 0,77 \\
\hline LABINT & $\begin{array}{c}-1,1 * \star * \\
(6,21)\end{array}$ & $\begin{array}{l}16,9^{\star \star \star *} \\
(12,05)\end{array}$ & $\begin{array}{r}-11,6 \\
(1,75)\end{array}$ & $\begin{array}{c}-4,0^{\star \star \star} \\
(4,71)\end{array}$ & $\begin{array}{l}0,9^{\star \star \star} \\
(4,02)\end{array}$ & $\begin{array}{r}-76,1 \text { * } \\
(2,56)\end{array}$ & 0,73 & 0,59 \\
\hline $\mathrm{MACH} \ldots \ldots \ldots \ldots$ & $\begin{array}{l}1,1^{* * *} \\
(4,95)\end{array}$ & $\begin{array}{r}4,8 \text { * } \\
(1,92)\end{array}$ & $\begin{array}{r}-10,0 \\
(1,07)\end{array}$ & $\begin{array}{c}-3,1 * * * \\
(6,57)\end{array}$ & $\begin{array}{l}0,1 \\
(0,81)\end{array}$ & $\begin{array}{r}-17,9 \\
(1,37)\end{array}$ & 0,91 & 0,87 \\
\hline CHEM ....... & $\begin{array}{c}-0,1 \\
(1,42)\end{array}$ & $\begin{array}{l}6,5 * * \star \\
(6,24)\end{array}$ & $\begin{array}{l}-6,6 \\
(1,74)\end{array}$ & $\begin{array}{r}-2,3 * * \\
(2,67)\end{array}$ & $\begin{array}{l}0,4^{*} \\
(1,88)\end{array}$ & $\begin{array}{c}-14,6 \\
(0,73)\end{array}$ & 0,69 & 0.53 \\
\hline
\end{tabular}

Notas: Estadísticos $\iota$ (entre paréntesis) ajustados para tener en cuenta la heterocedasticidad siguiendo el procedimiento de White (1980). Los valores críticos (contraste de dos colas) proceden de la distribución de la $t$ de Student con 12 grados de libertad.

* significativo al nivel de $10 \%$.

** significativo al nivel de $5 \%$.

$\star \star \star$ significativo al nivel de $1 \%$.

FUENTE: Véase el apéndice.

El cuadro 1.7 muestra los aranceles sobre las importaciones en porcentaje de las importaciones especiales totales de los dieciocho paises incluidos en este estudio y otros índices globales de protección relacionados con los bienes manufacturados y el trigo ${ }^{49}$. El periodo $1892-1914$ se caracterizó por un reforzamiento del proteccionismo en la Europa continental y en importantes socios comerciales europeos: Estados Unidos con el arancel McKinley de 1890, Australia con su legislación arancelaria de 1906, el arancel argentino de 1891 y Canadả con sus medidas proteccionistas adoptadas tras la aplicación en $1878 \mathrm{del}$ programa electoral de «política nacional». Gran Bretaña se mantuvo a la vanguardia del movimiento en favor del libre comercio después de 1846, aunque tenía unos aranceles relativamente altos sobre las bebidas alcohólicas, el té, las especias y el azúcar. Alemania desempeñó un papel fundamental en el retorno

49 El analisis siguiente de la politica comercial (1892-1914) sigue a Bairoch (1989). 
de Europa continental al proteccionismo a la vuelta de siglo con el nuevo arancel de 1902. Francia se volvió cada vez más proteccionista, primero con el doble arancel de 1892 y las numerosas revisiones arancelarias realizadas en los años posteriores. Austria-Hungria se encontraba a medio camino entre los países más liberales de Europa y los más proteccionistas. Italia mantuvo hasta justo antes de la Primera Guerra Mundial un arancel más liberal que la mayoria de los grandes paises del continente. España y Portugal siguieron siendo muy proteccionistas tras los aranceles establecidos en 1892. La adopción de una política comercial en los paises europeos más pequeños, que se basaban más en el comercio, fue más desigual que en los grandes. Los Paises Bajos y, hasta cierto punto, Suiza y Bélgica adoptaron una política liberal. Por lo que se refiere a los pequeños paises industrializados, Suecia siguió siendo indudablemente el pais más proteccionista. Los tres países escandinavos no industrializados (Dinamarca, Noruega y Finlandia), que seguían tradicionalmente una politica de libre comercio, vieron aumentar considerablemente sus aranceles sobre las importaciones en este periodo.

\section{CUADRO 1.7}

Algunos indicadores de los niveles de aranceles sobre las importaciones en 1913

\begin{tabular}{|c|c|c|c|}
\hline & $\begin{array}{c}\text { Aranceles sobre las } \\
\text { importaciones en } \% \\
\text { de las importaciones } \\
\text { totales especiales }\end{array}$ & $\begin{array}{l}\text { Nivel medio } \\
\text { aproximado de los } \\
\text { aranceles sobre } \\
\text { los productos } \\
\text { manufacturados }\end{array}$ & $\begin{array}{l}\text { Nivel de los } \\
\text { aranceles sobre } \\
\text { el trigo }\end{array}$ \\
\hline Argentina ......... & 21,6 & 28 & 0 \\
\hline Australia $\ldots \ldots \ldots \ldots \ldots \ldots \ldots$ & 18,2 & 16 & 22 \\
\hline Austria-Hungria $\ldots \ldots \ldots \ldots \ldots$ & 7,6 & 20 & 35 \\
\hline Bélgica .......................... & 15,8 & 9 & 0 \\
\hline Canadá $\ldots \ldots \ldots \ldots \ldots \ldots \ldots$ & 18,7 & 26 & - \\
\hline Dinamarca $\ldots \ldots \ldots \ldots \ldots \ldots \ldots$ & 5,8 & - & 0 \\
\hline Finlandia .. & 12,1 & 28 & 0 \\
\hline Francia ... & 8,7 & 21 & 38 \\
\hline Alemania & 7,9 & 13 & 36 \\
\hline Italia $\ldots \ldots \ldots \ldots \ldots \ldots \ldots$ & 9,7 & 20 & 40 \\
\hline Paises Bajos .................... & 0,4 & - & 0 \\
\hline Noruega $\ldots \ldots \ldots \ldots \ldots \ldots \ldots$ & 11,4 & - & 4 \\
\hline Portugal $\ldots \ldots \ldots \ldots \ldots \ldots \ldots$ & 23,7 & - & Prohibitivos \\
\hline España $\ldots \ldots \ldots \ldots \ldots \ldots \ldots$ & 14,3 & 34 & 43 \\
\hline Suecia $\ldots \ldots \ldots \ldots \ldots \ldots \ldots \ldots$ & 9,0 & 25 & 28 \\
\hline Suiza $\ldots \ldots \ldots \ldots \ldots \ldots \ldots \ldots \ldots$ & 4,4 & 8 & 2 \\
\hline Reino Unido .................. & 5,6 & 0 & 0 \\
\hline Estados Unidos . . . . . . . . . . . . . & 21,4 & 44 & 0 \\
\hline
\end{tabular}

Fuente: P. Bairoch (1989, p. 76). 
A. COMERCIO TOTAL
IAC $=-29,5+3,0$ Arancel
$R 2=0,55$
$(-1,3) \quad(1,8)$

B. COMERCIO DE BIENES MANUFACTURADOS
$\mathrm{IAC}=-30,8+1,9$ Arancel
$R 2=0,72$
$(-2,9) \quad(2,6)$

Notas: Véase el texto.

A falta de datos más desagregados sobre los aranceles y las exportaciones netas, en este apartado presentamos algunos datos sobre la exactitud del indice ajustado del comercio como medida de la distorsión provocada por la política comercial. Se realiza una regresión del índice ajustado del comercio deducido de la estimación de la ecuación 8 con respecto a una medida de los niveles arancelarios correspondientes a las exportaciones netas totales y al conjunto de bienes manufacturados. La medida de los aranceles que utilizamos aquí son los aranceles sobre las importaciones en porcentaje de las importaciones especiales totales (cuadro 1.7), ya que es la única medida que permite hacer comparaciones entre los dieciocho países de la muestra. El cuadro 1.8 presenta los resultados de este ejercicio ${ }^{50}$. La figura 1.1 muestra una relación visual del agregado de bienes manufacturados. Los países del Nuevo Mundo que tenían unos aranceles globales más altos (Argentina, Canadá, Australia y Estados Unidos) tienden a tener menos exportaciones netas que las que predice el modelo. En las pequeñas economías abiertas del Viejo Mundo (Países Bajos, Dinamarca, Suecia, Finlandia), las exportaciones netas son mayores de

so En el cuadro 18 se utiliza una muestra limitada que excluye a Suiza y Noruega, considerados atipicos. Aunque Noruega sigue la pauta esperada en el grupo de pequeñas economias abiertas que se encuentran en el cuadrante inferior izquierdo de la figura 1.1, el índice ajustado del comercio negativo es demasiado extremo. Suiza, por su parte, es una economía abierta que tiene unas exportaciones netas inesperadamente grandes en la industria manufacturera. En este caso, el modelo no es capaz de recoger los «accidentes históricos», como la pauta suiza de exportación de instrumentos profesionales altamente especializados. No deja de ser interesante el hecho de que también se observa esta misma conducta atípica en Suiza en los estudios modernos de la apertura (Leamer, 1988, p. 199). 
lo esperado. En futuras investigaciones veremos si este método para detectar las distorsiones provocadas por la política comecial puede aplicarse en un grado más desagregado ${ }^{51}$.

FIGURA 1.1

Aranceles e indice ajustado del comercio

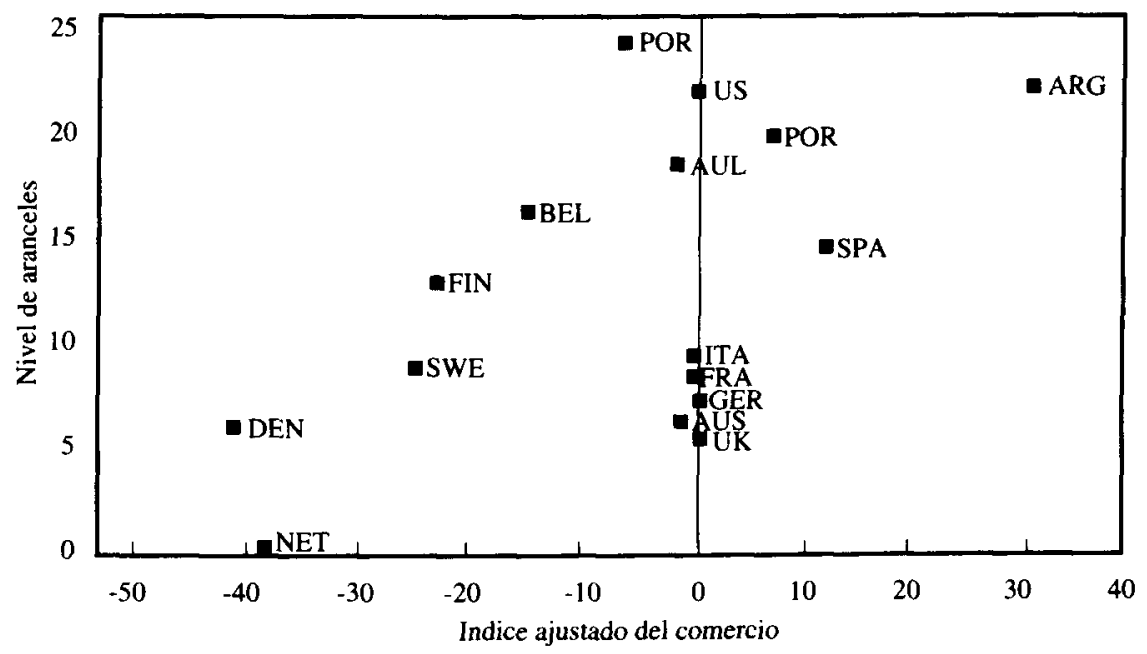

Fuente: Véase el texto.

\section{EXPLICACIÓN DE LAS DISTORSIONES PROVOCADAS POR LA POLITICA COMERCIAL: ALGUNAS HIPÓTESIS PROVISIONALES}

A continuación utilizamos el modelo estimado en el apartado $V$ para identificar los países atípicos. El cuadro 1.9 muestra los estadísticos $t$ de las variables ficticias de los países que se fueron introduciendo de una en una en cada regresión y el 1.10 resume las observaciones más extremas. El país que más se

51 Debe señalarse que el indice ajustado del comercio recoge las desviaciones de las exportaciones netas, mientras que las barreras comerciales sólo recogen las importaciones. Al analizar los residuos, deberia considerarse una interpretación más general de las distorsiones provocadas por la política comercial. Véase el apartado VI. 
aleja es España, seguido del Reino Unido, en todos los grupos de mercancías, salvo LABINT y CHEM. También parece que Italia es una observación atípica en AGPRO, RAWMAT y MACH. Otros países atipicos en determinados grupos de mercancias son Estados Unidos en CAPINT y CHEM; Alemania en CHEM; Suiza en LABINT, y los Paises Bajos en CAPINT. Parece que los dos casos más claros son Suiza y Alemania. La observación alemana confirma el papel de la dotación especial de este país en algunos productos clave para la industria química ${ }^{52}$ y una educación química científica inexistente en los demás paises. Las empresas químicas alemanas establecieron un monopolio de conocimientos que se mantuvo gracias a la existencia de una educación apropiada. Por otra parte, la industria química alemana era muy distinta de la de otros paises industrializados y se basaba mucho más en aplicaciones de la quimica orgánica, rama insignificante en otros países, salvo Suiza. En el caso de Suiza, el modelo no es capaz de recoger «accidentes históricos» como la pauta de exportación de instrumentos profesionales altamente especializados e intensivos en trabajo de este pais 53 .

Una posible explicación ad boc de la variable ficticia de los Paises Bajos son los errores de medición o la calidad de las estadisticas sobre el comercio. En esa época había, en términos generales, dos métodos de valoración: los valores oficiales y los valores declarados. El primero consiste en listas de valores de todas y cada una de las mercancías elaboradas por el gobierno y ajustadas de vez en vez cuando cambiaban las circunstancias y los precios. Este sistema tiene su origen en la incapacidad de los gobiernos para confiar en que los comerciantes declararan exactamente los bienes y los oficiales de aduanas no aceptaran sobornos para tolerar declaraciones falsas. Pero los valores oficiales pronto se alejaron de los reales debido a que se revisaban poco o a otras razones. Un notable y documentado caso es el de los Países Bajos hasta la Primera Guerra Mundial, en el cual las listas operativas de valores oficiales eran entre un 25 y un 40 por ciento superiores a los valores de mercado ${ }^{54}$. Como ha señalado Yates ${ }^{55}$, «no sé de ningún intento de ajustar los valores del comercio anterior a 1914 de los Paises Bajos, por lo que siguen siendo una partida indebidamente hinchada en las estadisticas mundiales».

52 Los más importantes son los depósitos de potasio de Stassfurt, que satisfacian las necesi dades mundiales hasta 1925.

"Como se señala en la nota 27 , también se observa la misma conducta atipica en el caso de Suiza en estudios modernos de la apertura (Leamer, 1988, p. 199).

54 Véase sobre todo P. L. Yates (1959) y G. Federico y A. Tena (1991).

ss Yates (1959), p. 26. 


\section{CUADRO 1.9}

El modelo de Heckscher y Oblin en 1913

(Estadísticos $t$ de las variables ficticias de los países, ecuación 9)

\begin{tabular}{|c|c|c|c|c|c|c|}
\hline & $A G P R O$ & $R A W M A T$ & CAPINT & $L A B I N T$ & $M A C H$ & CHEM \\
\hline Argentina & 1,7 & 0,0 & 0,2 & 1,4 & 0,5 & 0,5 \\
\hline Australia .. & $-1,9$ * & $-0,1$ & 0,0 & $-1,0$ & $-0,7$ & $-1,6$ \\
\hline Austria .. & 0,0 & $-0,5$ & $-0,4$ & 0,5 & 0,6 & 0,6 \\
\hline Bèlgica $\ldots \ldots \ldots \ldots \ldots$ & 0,2 & $-0,7$ & $-0,3$ & $-0,3$ & $-0,7$ & $-0,4$ \\
\hline Canadá............... & 0,3 & $-0,1$ & 0,7 & $-0,3$ & 0,6 & 1,6 \\
\hline Dinamarca ............. & 0,7 & 0,3 & $-0,5$ & $-0,4$ & 0,0 & 0,0 \\
\hline Finlandia .............. & 0,4 & 0,2 & $-0,2$ & $-0,1$ & 0,2 & 0,0 \\
\hline Francia $\ldots \ldots \ldots \ldots \ldots$ & $-1,0$ & 0,9 & 0,2 & 0,1 & $-1,4$ & $-0,2$ \\
\hline Alemania .............. & 1.5 & 0,1 & 1,0 & 1,7 & 1,1 & $5,7 \star \star \star$ \\
\hline Italia $\ldots \ldots \ldots \ldots \ldots \ldots$ & $1,8 *$ & $-2,7 * *$ & $-1,5$ & $-0,7$ & $2,6^{* *}$ & 0,1 \\
\hline Paises Bajos ............ & 0,2 & $-0,1$ & $-1,9 *$ & $-0,7$ & $-0,8$ & $-0,5$ \\
\hline Noruega............ & $-0,3$ & 0,2 & $-0,3$ & $-1,4$ & $-0,5$ & $-0,3$ \\
\hline Portugal ................ & 0,3 & $-0,3$ & $-0,3$ & 0,1 & 0,6 & 0,4 \\
\hline España $\ldots \ldots \ldots \ldots \ldots$ & $-2,9 * *$ & $4,2^{\star * *}$ & $2,7 * *$ & 0,5 & $-3,6 * * *$ & $-0,4$ \\
\hline Suecia . & 0,0 & 0,7 & $-0,2$ & $-0,8$ & 0,0 & $-0,7$ \\
\hline Suiza $\ldots \ldots \ldots \ldots \ldots$ & $-1,5$ & $-1,8 *$ & 1,7 & 3,0 ** & 1,3 & $-1,7$ \\
\hline Reino Unido ........... & $-3,3 * * *$ & $3,9 * \star *$ & 2,3 ** & $-0,5$ & $-2,7^{\star *}$ & $-0,7$ \\
\hline Estados Unidos ........ & 0,3 & $-1,7$ & $-2,2 * *$ & $-0,9$ & 0,5 & $-2,6 * \star$ \\
\hline
\end{tabular}

Notas: El valor de la $t$ se refiere a la variable ficticia obtenida seleccionando en cada ecuación un pais de cada vez. Los valores críticos (contraste de dos colas) proceden de la distribución de la $t$ de Student con 12 grados de libertad.

* significativo al nivel de $10 \%$.

** significativo al nivel de $5 \%$.

*** significativo al nivel de $1 \%$.

Fuente: Véase el apéndice.

\section{La pauta que muestran España e Italia, asi como el Reino Unido y Estados} Unidos ${ }^{56}$, necesita un contraste más desagregado para distinguir los factores

56 Una posible explicación de la conducta atípica del Reino Unido podría ser, al igual que en el caso de los Paises Bajos, el sistema de presentación de la información que se utiliza en las estadisticas del comercio. El Reino Unido y los Países Bajos eran los mayores reexportadores en este periodo. Mientras que el Reino Unido publicaba un análisis de «reexportación», no ocurria asi con los Paises Bajos. En este sistema de contabilidad del comercio, las exportaciones netas tienden a estar sesgadas a la baja, ya que el pais registra todas sus importaciones, pero sólo una parte de sus exportaciones. Por otro lado, este método no sólo sesga a la baja la medida de las exportaciones netas de un pais, sino que tambièn sesga a la baja las exportaciones netas de este pais en relación con los que utilizan un sistema general «completo» (exportaciones nacionales más reexportaciones). En el caso del Reino Unido, las estimaciones del Annual Statement of Trade (1914) sugieren que las reexportaciones eran, en realidad, muy importantes (en concreto, el 
que explican la conducta específica de cada pais. El cuadro 1.1 muestra los valores que tenían en 1913 las importaciones y las exportaciones de los seis sectores de las dos economías sureñas. España era un exportador neto de AGPRO, RAWMAT y LABINT y un importador neto de CAPINT, MACH y CHEM, como era de esperar en una economía atrasada. Italia sólo era un exportador neto de bienes CAPINT y un importador neto de todo lo demás, pauta que refleja la expansión industrial de finales del periodo giolittiano. Según el cuadro 1.10, ni España ni Italia tenían una pauta comercial excepcional en el caso de los bienes LABINT y CHEM, de acuerdo con el modelo. Sin embargo, en el resto su pauta de exportaciones netas se alejaba de lo que sería de predecir, con la excepción de CAPINT en el caso de Italia. El resultado es interesante debido al sentido opuesto de estas desviaciones de la «norma». El modelo predice en el caso de España un superávit mayor en el caso de AGPRO y un déficit mucho menor en el de MACH. Por otra parte, el valor de las exportaciones netas de RAWMAT parece demasiado alto y el déficit de CAPINT demasiado bajo. En cambio, Italia tiene unos déficit menores de lo esperado en el caso de AGPRO y MACH y mayores en el caso de RAWMAT.

La pauta comercial presentada en el cuadro 1.11 refleja una estructura de dotaciones diferente en el caso de España e Italia. Como señala Clive Trebilcock, «España e Italia se encuentran, pues, en puntos recíprocos de la escala tanto de dotación de recursos como de utilización de los recursos: Italia está muy mal dotada, pero supo sobreponerse a la explotación extranjera para sacar el mayor rendimiento de lo que tiene; España tiene abundantes recursos naturales pero carece del talento empresarial para capitalizar su activo antes de despilfarrarlo» ${ }^{57}$. Las reservas de carbón eran tan escasas en 1913 que Italia sólo podía extraer $1 / 400^{\text {avo }}$ de la producción británica. Al ser el combustible principal en la era de la máquina de vapor, tenía que importar la mayor parte ${ }^{58}$. Poseia importantes depósitos de azufre en Sicilia, algún mineral de hierro en Val d'Aosta, en Terni (Umbría) y en la isla de Elba. La dotación de minerales de España era muy diferente. Había hierro de buena calidad en Murcia, Almeria, Santander y Vizcaya; plomo en las Sierras de Gador, Almagrera y Carta-

café, el té, el algodón en rama, la lana en bruto, los aceites, las semillas oleaginosas, las maderas de tinte y las materias colorantes, los cueros, las pieles, etc.). Los datos sobre el comercio utilizados en este artículo se basan en los datos publicados en Yates (1959) y Maizels (1938) tal como se describe en el apéndice. Ambos autores sostienen que trataron de tener en cuenta este sesgo estadístico, aunque no dan los detalles. Las investigaciones que están realizándose actualmente y que se basan en datos originales y más desagregados tratarán de explicar este problema estadístico.

57 Clive Trebilcock (1981), p. 308.

58 En 1913 el carbón se había convertido en el principal artículo de importación, seguido del algodón en rama. 
gena y en la parte central del sur; extensas reservas de cobre en Río Tinto y Tarsis; y las importantes minas de mercurio de Almadén. En Asturias y Córdoba había abundante carbón. Como señala Trebilcock, «la producción española de plomo en bruto sucedió a la británica y precedió a la americana en la dominación de los mercados mundiales en el periodo 1896-98; asimismo, el área de Huelva en su año de mayor auge (1912) representaba el 66 por ciento de la producción mundial de piritas y su mina más rica, Rio Tinto, el 44 por ciento. Las diferencias en lo que se refiere a la tierra productiva también eran importantes. En Italia, la tierra era casi toda ella yerma; según el estudio de la Sociedad de Naciones (1927), sólo el 66 por ciento de la superficie era cultivable. En el caso de España, el porcentaje de tierra agricola superaba el 80 por ciento. Por último, la medida de las cualificaciones utilizada en este estudio, la tasa de analfabetismo, muestra un revelador contraste entre los dos países: el 52,2 por ciento en España y el 37,2 por ciento en Italia. Lo que hay que explicar son las desviaciones de la pauta comercial que predice el modelo basado en las dotaciones. La hipótesis que presentamos aquí y que necesita un análisis más profundo basada en datos desagregados es que las desviaciones deben atribuirse a las distorsiones provocadas por la política comercial e industrial. En suma, parece que Italia debió de proteger en relación con la media mundial AGPRO y MACH, mientras que España protegió RAWMAT y CAPINT.

CUADRO 1.10

Países extremos 1913

\begin{tabular}{|c|c|c|}
\hline & $\begin{array}{c}\text { Exportaciones netas } \\
\text { reales superiores } \\
\text { a las predichas }\end{array}$ & $\begin{array}{l}\text { Exportaciones netas } \\
\text { reales inferiores } \\
\text { a las predichas }\end{array}$ \\
\hline AGPRO & Italia $(1,8)$ & $\begin{array}{l}\text { Reino Unido }(-3,3) \\
\text { España }(-2,9) \\
\text { Australia }(-1,9)\end{array}$ \\
\hline RAWMAT & $\begin{array}{l}\text { España }(4,2) \\
\text { Reino Unido }(3,9)\end{array}$ & Italia $(-2,7)$ \\
\hline CAPINT & $\begin{array}{l}\text { España }(2,7) \\
\text { Reino Unido }(2,3)\end{array}$ & $\begin{array}{l}\text { Estados Unidos }(-2,2) \\
\text { Países Bajos }(-1,9)\end{array}$ \\
\hline LABINT & Suiza $(3,0)$ & - \\
\hline $\mathrm{MACH}$ & Italia $(2,6)$ & $\begin{array}{l}\text { España }(-3,6) \\
\text { Reino Unido }(-2,7)\end{array}$ \\
\hline CHEM & Alemania $(5,7)$ & Estados Unidos $(-2,6)$ \\
\hline
\end{tabular}

FUENTE: A partir del cuadro 1.9, el valor de la $t$ corresponde a la variable ficticia que selecciona el pais. 


\section{CUADRO 1.11}

Valor de las exportaciones $y$ las importaciones por grupos de mercancías 1913: Italia y España

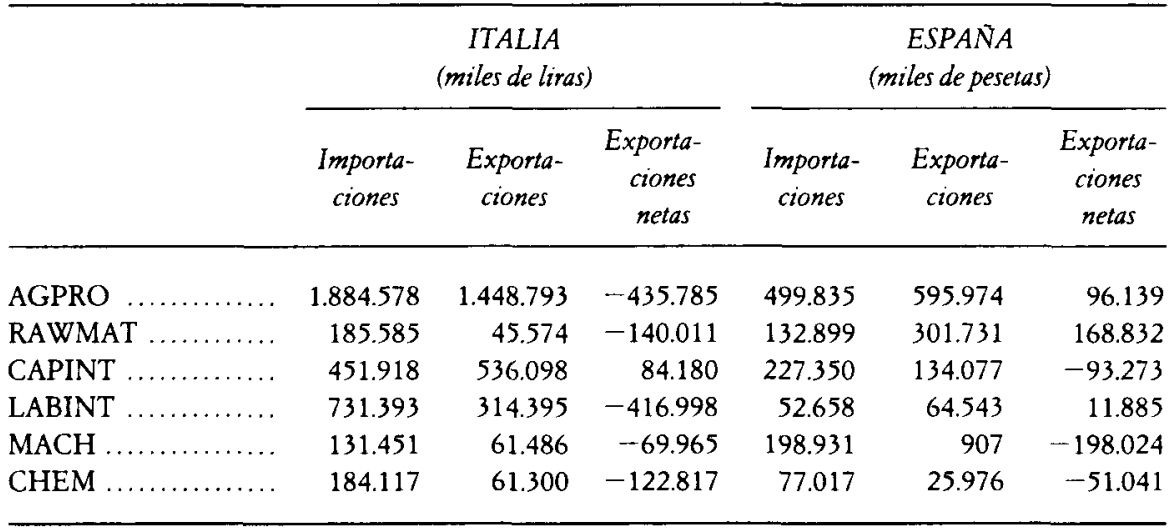

Notas: Véase el cuadro $2 \mathrm{~b}$ para las definiciones.

Fuentes: Véase el apéndice.

\section{OBSERVACIONES FINALES}

El modelo de comercio de Heckscher, Ohlin y Vanek es el eje central de la mayoría de los estudios empíricos sobre el comercio desde la Segunda Guerra Mundial. Implica que el vector de exportaciones netas es una función lineal del vector de las dotaciones de factores. También implica que todos los países tienen los mismos precios relativos de los factores (igualación de los precios de los factores). La proposición de $\mathrm{H}-\mathrm{O}$ se ha puesto en cuestión desde que se obtuvieron dos grandes resultados empíricos. El primero fue el descubrimiento de Leontief 59 de que las importaciones americanas eran más intensivas en capital que sus exportaciones. El segundo fueron las numerosas pruebas de la existencia de un comercio «intrasectorial» ${ }^{0}$. Desde entonces, la respuesta de los economistas ha sido en gran parte teórica, alterando la versión original del modelo o introduciendo nuevos modelos con rendimientos crecientes de escala y diferenciación del producto. En este artículo mantenemos que el modelo de comercio de Heckscher y Ohlin es absolutamente adecuado

\footnotetext{
59 Leontief (1953).

60 Grubel y Lloyd (1975).
} 
para analizar los últimos años del siglo XIX, periodo que motivó los escritos de Eli F. Heckscher y Bertil Ohlin justo después de la Primera Guerra Mundial.

Con el conjunto de datos de 1913 elaborado recientemente, en el apartado IV de este artículo observamos la existencia de poderosas pruebas que confirman la primera implicación de la versión del modelo de Heckscher, Ohlin y Vanek, a saber, que las dotaciones de factores explican los principales aspectos de la pauta de comercio observada antes de la Primera Guerra Mundial. La segunda implicación del modelo (igualación de precios de los factores) es coherente con los recientes trabajos de Williamson-O'Rourke y Williamson ${ }^{61}$. Al mismo tiempo, como señalamos en el apartado $\mathrm{V}$, el modelo demuestra ser un útil marco para analizar la política comercial distorsionadora de este periodo. Los resultados de estos dos apartados son preliminares y aguardan un análisis más desagregado.

\section{APENDICE: FUENTES ESTADISTICAS}

\section{Medida del stock de capital (CAP)}

Se ha utilizado el consumo de energía como variable que mide de forma aproximada el stock de capital. Se refiere al consumo aparente (producción más importaciones netas) de combustibles sólidos (carbón de antracita, lignito y coque). Para poder hacer agregaciones y comparaciones internacionales, los datos se expresan en miles de equivalentes de carbón de antracita (una tonelada métrica de carbón de antracita contiene aproximadamente 28 millones de btu --unidad de calor británicay es el calor necesario para calentar una libra de agua un grado Fahrenheit a la temperatura de máxima densidad o cerca de ella). Los factores de conversión utilizados en este cálculo proceden de J. Darmstadter (1971, p. 828). La fuente principal es B. R. Mitchell $(1980,1983)$. Sus datos se han contrastado minuciosamente y complementado con varios números de The Mineral Industry, compilado por R. P. Rothwell $(1913,1917$ y 1920). Siempre que había una discrepancia se ha utilizado la segunda fuente.

\section{Trabajo cualificado (SKI)}

El trabajo cualificado se obtiene multiplicando la población activa por la tasa de alfabetización. Los datos se expresan en miles de trabajadores.

61 Williamson (1992) y O'Rourke y Williamson (1992). 


\section{Trabajo no cualificado (UNSK)}

El trabajo no cualificado se obtiene restando de la población activa el trabajo no cualificado. Los datos se expresan en miles de trabajadores.

\section{Población activa}

Los datos de todos los países, salvo Argentina, España y Portugal, se refieren a la población activa total (en miles) estimada a mediados de año y proceden de A. Maddison (1982). Los datos de Argentina, Portugal y España se refieren a la población económicamente activa publicados en Mitchell $(1980,1982)$. Cuando ha sido necesario se ha utilizado una interpolación lineal entre los años censales.

\section{Tasa de analfabetismo}

La tasa de analfabetismo es, siempre que es posible, el porcentaje de la población de 10 años o más que no sabe leer y escribir. Los datos proceden de Abel y Bond (1929), Adelman y Morris (1984), Cipolla (1969), Dominion Bureau of Statistics (1926), Lindergren (1945), Sothi (1966), Stedman y Kaestle (1987) y UNESCO (1953, 1957).

\section{Tierra agricola $(A G R)$}

Existen datos sobre la tierra agricola en miles de hectáreas de todos los países (salvo Portugal) en Sociedad de Naciones (1926). En el caso de Portugal, los datos proceden de Lains (1989).

\section{Recursos minerales (MIN)}

Esta variable de recursos naturales es el valor en miles de dólares de la producción de petróleo más la producción de un compuesto de 12 minerales: bauxita, cobre, mineral de hierro, plomo, manganeso, níquel, fosfato, potasio, piritas, azufre, estaño y zinc. Los valores están expresados en miles de dólares. Las principales fuentes de la producción de todos los países son Mitchell $(1980,1983)$ y Rothwell (comp.), The Mineral Industry (varios números). Las fuentes secundarias de paises especificos son las siguientes:

Argentina

Australia

Bélgica

Canadá

Francia

Alemania

\section{Estadística Minera de la República}

Australian Mineral Industry: Production and Trade, 1842-1964

Statistique des Industries Extractives et Metallurgiques

Annual Report of the Mineral Production of Canada

Statistique de IIndustrie Minerale en France

Statisticbes Jabrbuch fur das Deutsche Reich.

Annual Summary of Metal Statistics by the Metallgesellschaft, Frankfurtam-Main 
Italia Rivista del Servizio Minerario

España Estadistica Minera de España

Reino Unido Annual Report of the Secretary for Mines

Estados Unidos Mineral Resources of the United States

Los precios proceden de Potter y Christy (1962), The Mineral Industry (varios números) y The Mining Magazine (varios numeros).

\section{Producto nacional (PNB)}

Todas las cifras están expresadas en millones de dólares americanos a precios corrientes convertidos a tipos de cambio de mercado.

Argentina PIB, procede de IEERAL (1986, cuadro 12).

Australia $\quad$ PIB, procede de Mitchell (1983, cuadro K1).

Austria PNB, procede de Mitchell (1980, cuadro J1).

Bélgica PNN, procede de Mitchell (1980, cuadro J1).

Canadá $\quad$ PNB (1910), procede de Mitchell (1983, cuadro K1).

Dinamarca $\quad$ PNB, procede de Mitchell (1980, cuadro J1).

Finlandia $\quad$ PIB, procede de R. Hjerppe y E. Pihkala (1977, pp. 59-68).

Francia PIB, procede de M. Levy-Leboyer y F. Bourguignon (1985, cuadro A III).

Alemania $\quad$ PNN, procede de Mitchell (1980, cuadro J1).

Italia PNB, procede de Mitchell (1980, cuadro J1).

Paises Bajos $\quad$ PNN, procede de Mitchell (1980, cuadro J1).

Noruega $\quad$ PIB, procede de Mitchell (1980, cuadro J1).

Portugal PNB, procede de D. Justino (1987, pp. 451-61).

España PNN, procede de Mitchell (1980, cuadro J1).

Suecia PIB, procede de Mitchell (1980, cuadro J1).

Suiza PNN, procede de Mitchell (1980, cuadro J1).

Reino Unido PNB, procede de Mitchell (1980, cuadro J1).

Estados Unidos PNB, procede de Mitchell (1983, cuadro K1).

NoTA: PIB: producto interior bruto; PNB: producto nacional bruto; PNN: renta nacional neta.

\section{Tipos de cambio}

Los tipos de cambio utilizados para convertir las monedas nacionales en dólares americanos proceden de Board of Governors of the Federal Reserve System (1943), salvo en el caso de los siguientes países:

Argentina IEERAL (1986, p. 124).

Australia G. A. Rousch (1914, pp. x-xi). 
Finlandia Sociedad de Naciones (1927).

Noruega G. A. Rousch (1914, pp. x-xi).

Portugal G. A. Rousch (1914, pp. x-xi).

España A. Carreras, comp. (1989, cuadro 99).

\section{Datos sobre las exportaciones netas}

Los datos sobre el comercio se han agregado en seis grupos de mercancías comparables a los agrupamientos realizados por Leamer (1984). Se expresan en miles de dólares a tipos de cambio de mercado. Las mercancías se han clasificado de acuerdo con la Clasificación Internacional Uniforme del Comercio (revisada en 1961) a dos dígitos (véase el cuadro 2b). Los datos se expresan en miles de dólares. Los agregados de Canadá, Francia, Italia, Suecia, Suiza, Reino Unido y Estados Unidos se han realizado utilizando principalmente dos fuentes de datos - P. Lamartine Yates (1959) y Alfred Maizels (1963) _ de la siguiente manera:

AGPRO Comprende los «productos alimentarios» y los «productos agricolas» de Yates (1959).

RAWMAT Comprende los «minerales totales» de Yates (1959) y los «metales» de Maizels (1963).

CAPINT Comprende los «bienes metálicos», los «textiles y confección: hilaturas», los «textiles y confección: tejidos» y «otros bienes manufacturados: intermedios» de Maizels (1963).

LABINT Comprende «otros bienes manufacturados: bienes acabados» y los «textiles y confección: bienes fabricados» de Maizels (1963).

$\mathrm{MACH} \quad$ Comprende la «maquinaria» y el «material de transporte» de Maizels (1963).

CHEM Comprende los «productos químicos: intermedios y acabados» de Maizels (1963).

Los agregados de Argentina, Australia, Austria-Hungría, Bélgica, Dinamarca, Finlandia, Alemania, Paises Bajos, Noruega, Portugal y España se han elaborado utilizando principalmente la fuente de Sociedades de Naciones (1928), Memorandum on International Trade and Balance of Payments 1912-1926. Todos los articulos se han codificado utilizando la Clasificación Internacional Uniforme del Comercio revisada (1961) a 2 digitos y se han agregado de acuerdo con el cuadro $2 \mathrm{~b}$. Ha sido necesario realizar algunos ajustes para que los datos de los dos grupos de paises fueran comparables y para tener en cuenta las mercancias no clasificadas que no aparecen en el memorándum de la Sociedad de Naciones de 1928, para lo cual se han utilizado los datos de Estevadeordal (1993) que emplea datos sobre el comercio desagregados a 2 dígitos. 


\section{BIBLIOGRAFÍA}

Abel, J. E., y Bond, N. J. (1929): Illiteracy in Several Countries of the World, United States Bureau of Education.

Adelman, I., y C. T. Morris (1984): Comparative Patterns of Economic Development 18501914, Baltimore, The Johns Hopkins University Press.

Allen, R. C. (1979): «International Competition in Iron and Stell, 1850-1913», Journal of Economic History, vol. 39, pp. 911-937.

Balroch, P. (1989): «European Trade Policy, 1815-1914», pp. 1-160 en P. Mathias y S. Pollard (comps.), The Cambridge Economic History of Europe, Cambridge, Cambridge University Press, vol. III.

BALASSA, B. (1965): «Trade Liberalization and 'Revealed' Comparative Advantage», The Mancbester School, vol. 33, pp. 99.123.

BOARD OF GOVERNORS OF THE FEDERAL System (1943): Banking and Monetary Statistics 1914-1941, septiembre, Washington, D. C.

BOARD OF TRADE (1904): British and Foreign Trade and Industrial Conditions, Londres.

BOWEN, H. P. (1983): «Changes in the International Distribution of Resources and their Impact on U. S. Comparative Advantage», Review of Economic and Statistics, vol. LXV, pp. 402-414.

Carreras, A. (comp.) (1989): Estadísticas Históricas de España. siglo XIX-XX, Madrid, Fundación Banco Exterior.

Caves, R. E.; J. A. Frankel y R. W. Jones (1990): World Trade and Payments, Harper CoIlins Publishers, $5^{\mathrm{a}}$ ed.

Cipol.LA, C. M. (1969): Literacy and Development in the West, apéndice estadístico, Londres, Pinguin Books Ltd.

Crafts, N. F. R. (1989): «Revealed Comparative Advantage in Manufacturing, 1899. 1950», Journal of European Economic History, vol. 18, núm. 1, pp. 127-137.

CrafTS, N. F. R., y M. Thomas (1986): «Comparative Advantage in UK Manufacturing Trade, 1910-1935m, The Economic Journal, vol. 96, pp. 629-645.

DarmstadTER, J. (1971): Energy in the World Economy, Baltimore, The Johns Hopkins Press.

DeArdorff, A. V. (1984): «Testing Theories and Predicting Trade Flows», en R. W. Jo. NES y P. B. KENEN (comps.), Handbook of International Economics, vol. I, pp. 467-517, Elsevier Science Publishers.

DePaRTMENT OF Mines (1913): Preliminary Report on the Mineral Production of Canada during the Calendar Year 1912, Ottawa, Goverment Printing Bureau.

Dixir, A. K., y V. D. Norman (1980): Theory of International Trade, Cambridge, Cambridge University Press.

Dominion Burfau of Statistics (1926): Illiteracy and School Attendance in Canada, Ottawa, capitulo 3.

Estevadeordal, A. (1992): «Comparative Advantaje at the Turn of the Century», Cambridge, Department of Economics, Harvard University, multicopiado. (1993a): «The Evolution of Comparative Advantage from 1913 to 1938», Cambridge, Department of Economics, Harvard University, multicopiado.

(1993b):, «Measuring Trade Openness in the Late Nineteenth Century: A HeckscherOhlin Approach», Cambridge, Department of Economics, Harvard University, multicopiado. 
FAO (1958): Production Yearbook, Ginebra.

FitzPatrick, G. L., y M. J. Modlin (1986): Direct-Line Distances. International Edition, Metuchen, The Scarecrow Press, Inc.

Flam, H., y M. J. Flanders (comps.) (1991): Heckscher-Oblin Trade Theory, Cambridge, The MIT Press.

Fraile, P. (1992): «Los mercados del centro y las economias atrasadas de Europa 19001930 m, en L. PRAdOS DE la Escosura y V. ZamaGni (eds.), El desarrollo económico en la Europa del Sur: España e Italia en perspectiva bistórica, Madrid, Alianza Universidad, pp. $253-268$.

FRANK, A. G. (1959): «Industrial Capital Stocks and Energy Consumption», The Economic Journal, vol. LXIX, pp. 170-174.

HAWKE, G. R. 81975): «The United States Tariff and Industrial Protection in the Late Nineteenth Century», Economic History Review, nüm. 28, pp. 84-99.

Hjerppe, R., y E. Pihkala (1977): «The Gross Domestic Product of Finland in 18601913. A preliminary estimate», Economy and History, vol. XX, núm. 2, pp. 59-68.

IEERAL (Instituto de Estudios Económicos sobre la Realidad Argentina y Latinoamericana) (1986): «Estadísticas de la evolución económica argentina 1913-1984», Estudios, núm. 9, julio/septiembre, pp. 103-184.

LaINs, P. (1989): «Foreign Trade and Economic Growth in the European Periphery. Portugal, 1850-1913», Florencia, European University Institute, multicopiado.

Lawrence, R. Z. (1987): «Does Japan Import Too Little: Closed Minds or Markets», Brookings Papers in Economic Activity, núm. 2, pp. 517-554.

Leamer, E. E. (1974): «The Commodity Composition of International Trade in Manufactures: An Empirical Analysis», Oxford Economic Papers, vol. 26, pp. 350-374. (1984): Sources of International Comparative Advantage, Cambridge, The MIT Press. (1988): «Measures of Openness», en R. E. Baldwin (comps.), Trade Policy Issues and Empirical Analysis, Chicago, The University of Chicago Press, pp. 147-204.

LeAmer, E. E. y H. P. Bowen (1981): «Cross-Section Tests of the Heckscher-Ohlin Theorem: Comment», American Economic Review, vol. 71, pp. 1040-1043.

Leontief, W. W. (1953): «Domestic Production and Foreign Trade: The American Capital Position Re-examined», Proceedings of the American Pbilosopbical Society, pp. 332-349.

Levy-Leboyer, M., y F. Bourguignon (1985): LEconomie Française au XIX siècle, París, Economica.

Liepmann, H. (1938): Tariff Levels and The Economic Unity of Europe, Londres, George Allen \& Unwin Ltd.

LINDERGREN, A. M. (1945): «Literacy and Illiteracy in Various Countries of the World», Education for Victory, vol. 3, núm. 21.

Maddala, G. S. (1977): Econometrics, Nueva York, McGraw-Hill.

Maddison, A. (1982): Phases of Capitalist Development, Oxford, Oxford University Press.

MaIzels, A. (1963): Industrial Growth and World Trade, Cambridge, Cambridge University Press.

Milward, A. S., y S. B. SAUl (1977): The Development of the Economies of Continental Europe 1850-1914, Cambridge, Cambridge University Press.

Ministerio de Agricultura (1911): Estadística Minera de La República 1909, Sección Geología, Mineralogia y Mineria, vol. 3, nüm. 3, Buenos Aires.

Mitchell, B. R. (1980): European Historical Statistics 1750-1975, The MacMillan Press, $2^{\mathrm{a}}$ ed. 
(1983): International Historical Statistics: The Americas and Australasia, The MacMillan Press.

Molinas, C., y L. Prados de la Escosura (1989): “Was Spain Different? Spanish Historical Backwardness Revisited», Explorations in Economic History, núm. 26, pp. 385 402.

Mulhall, M. G. (1896): Industries and Wealth of Nations, Londres, Longmans and Co.

- (1899), Dictionary of Statistics, Londres, George Rotledge and Sons, Ltd., $4^{\mathrm{a}} \mathrm{ed}$.

OIT (Organización Internacional del Trabajo) $(1949,1955)$ : Yearbook of Labor Statistics, Ginebra.

O'Rourke, K., y J. G. Williamson (1992): «Were Heckscher and Ohlin Right? Putting History Back into the Factor-Price-Equalization Theorem», Discussion paper number 1593, Cambridge, Harvard Institute of Economic Research.

O'Rourke, K., A. M. Taylor y J. G. Williamsom (1993): «Land, Labor and The WageRental Ratio Factor Price Convergence in the Late Nineteenth Century», Discusson Paper Number 1629, Cambridge, Harvard Institute of Economic Research.

Pollakd, S. (1981): Peaceful Conquest. The Industrialization of Europe 1760-1970, Oxford, Oxford University Press.

Potter, N., y F. T. Christy, Jr. (1962): Trends in Natural Resource Commodities, Baltimore, Johns Hopkins Press.

PRITCHETT, L. (1991): «Measuring Outward Orientation in Developing Countries», PER Working Paper no. 566, Banco Mundial.

Rothwell, R. P. (comp.): The Mineral Industry, Its Statistics, Tecbnology and Trade, Nueva York, Scientific Publishing Company, varios números.

Rousch, G. A. (comp): The Mineral Industry, Its Statistics, Technology and Trade, Nueva York, Scientific Publishing Company, varios números.

SAUL, S. B. (1965): «The Export Economy, 1870-1914», Yorkshire Bulletin of Economic and Social Research, vol. XVII, núm. 1.

SAxonhouse, G. R. (1983): «The Micro- and Macroeconomics of Foreign Sales to Japan», en W. R. Cline (comp.), Trade Policy for the 1980's, Cambridge, MIT Press.

(1986): «What's wrong with Japanese Trade Structure», Pacific Economic Papers, núm. 137, pp. 1-36.

SOCiEdAd DE NACiones (1927a): Population and Natural Resources, Ginebra. (1927b): Tariff Level Indices, Ginebra.

- (1927c): Memorandum on Balance of Payments and Foreign Trade 1911-1925. Volumen II. Trade Statistics, Ginebra.

SoTHI, P. (1966): Trends of World Illiteracy since 1900 and its Relationships with some Selected Educational, Social and Economic Factors, tesis doctoral, Ann Arbor, University of Michigan.

Stedman, L. C., y C. F. Kaestle (1987): «Literacy and Reading Performance in the United States. From 1880 to Present», Reading Research Quarterly, vol. 22, núm. 1.

TENA, A. (1992): «Protección y competitividad en España e Italia, 1890-1960», en L. Prados de la Escosura y V. ZamaGni (eds.), El desarrollo económico en la Europa del Sur: España e Italia en perspectiva bistórica, Madrid, Alianza Universidad.

Toniolo, G. (1977): «Effective Protection and Industrial Growth: The Case of Italian Engineering, 1898-1913», Journal of European Economic History, vol. 6, núm. 3, pp. 659-673. 
Tortella, G. (1990): «Education and Economic Development since the Industrial Revolution: A Summary Report», en H. VAN DER WEE y E. AERTS (comps.), Debates and Controversies in Economic History, Leuven, Leuven University Press.

TREBILCOCK, C. (1981): The Industrialization of the Continental Powers 1780-1914, Nueva York, Longman Group Ltd.

TYsZYNSKI, H. 1951): «World Trade in Manufactured Commodities, 1899-1950», The Manchester School, vol. 19, pp. 272-304.

UNESCO (1953), Progress of Literacy in Various Countries, Paris.

- (1957): World Illiteracy at Mid-Century, Paris.

WhITE, H. (1980): «A Heteroskedasticity-Consistent Covariance Matrix Estimator and a Direct Test for Heteroskedasticity», Econometrica, vol. 48, pp. 817-38.

Williamson, J. G. (1992): «The Evolution of Global Markets in the First and Second World Since 1830: Background evidence and Hypotheses», Working Paper no. 36, Cambridge, National Bureau of Economic Research (NBER/DAE).

WOYTINSKY, W. (1926): Die Welt in Zablen, Berlin, vol. IV.

WRIGHT, G. (1990): «The Origins of American Industrial Success, 1879-1940», American Economic Review, vol. 8, pp. 651-668.

Yates, P. Lamartine (1959): Forty Years of Foreign Trade, Londres, George Allen \& Un win Ltd. 